Bull. Mater. Sci., Vol. 6, No. 3, July 1984, pp. 417-452. (C) Printed in India.

\title{
High resolution electron microscopy of long range ordered alloys
}

\author{
S AMELINCKX ${ }^{\dagger}$, G VAN TENDELOO and J VAN LANDUYT \\ Rijksuniversitair Centrum Antwerpen, Groenenborgerlaan 171, B 2000-Antwerpen, Belgium \\ ${ }^{\dagger}$ Also at: SCK, B 2400-MOL, Belgium
}

\begin{abstract}
In this paper a survey is given of the application of high resolution electron microscopy and selected area electron diffraction to the study of long range ordered alloys. The characteristic features of the diffraction effects and of the imaging of ordered alloys are discussed. Group theoretical considerations allow prediction of microtexture of such alloys.

A number of simple alloy structures are imaged and it is shown, by means of simulated images, that under suitable diffraction conditions the columns of minority atoms are represented as bright dots. The columns of majority atoms are only visible at very small thicknesses. Subsequently a number of one- and two-dimensional long period superstructures derived from these simple structures are analysed, using high resolution micrographs. Finally the observed microtexture of the same alloy superstructures is compared with the predictions of group theory. Strongly non-conservative antiphase boundaries are found to dissociate in APB's which are as close to conservative as is compatible with the FCC lattice.
\end{abstract}

Keywords. High resolution electron microscopy; long range order; superstructure; modulated structure; antiphase boundries; domain structures.

\section{Introduction}

Single crystals of long range ordered alloys are usually highly fragmented in a large number of symmetry-related domains. The x-ray diffraction pattern of such a "single" crystal consists of the superposition of diffraction patterns due to all orientation variants. In structures with low symmetry the number of orientation variants may be as large as 12 or even 24. It is then in general difficult, if not impossible, to unscramble such a pattern into single domain patterns. Usually one can therefore only use $x$-ray powder diffraction methods with their well known limitations to determine the structures of the ordered alloys.

On the other hand when using selected area electron diffraction, combined with high resolution electron microscopy one can often select a single domain, as seen on the image, and obtain a monodomain diffraction pattern from it. In cases where the domain size is too small, even for selected area electron diffraction, one can sometimes obtain useful information from the optical diffraction pattern of a single domain on a high resolution image.

Long period interface modulated superstructures derived from simpler basic superstructures produce diffraction patterns which have characteristic geometrical features, which allow determination of modulated structure provided the basic structure can be identified.

Whereas $\mathrm{x}$-ray diffraction is the most suitable method to determine the basic structure, electron diffraction, assisted by high resolution electron microscopy, is the most powerful technique to determine modulated structures. We shall illustrate this by 
means of a number of case studies of long range ordered alloys and their modulated derivatives (Amelinckx 1978-79).

\section{Diffraction effects due to translation interface modulated structures}

Structures which are derived from a basic structure by the periodic insertion of identical parallel translation interfaces, produce characteristic diffraction effects which we shall now discuss. Let $\bar{R}$ be the displacement vector of the parallel interfaces, $d$ their distance and $\bar{n}$ their unit normal. The introduction of these interfaces produces a long period superstructure (figure 1). Let $\bar{g}$ represent vectors of the reciprocal lattice of the basic structure and $\bar{H}$ those of the superstructures, it was then shown (Amelinckx 1979, De Ridder et al 1972) that

$$
\bar{H}=\bar{g}+\frac{1}{d}(m-\bar{g} \cdot \bar{R}) \bar{n},
$$

where $m$ is a (small) integer. This expression proves that the diffraction pattern of the superstructures consists of linear sequences of equidistant spots (one for each value of $m$ ) associated with each basic spot $\bar{g}$. The spots within a sequence are separated by a distance $1 / d$, and furthermore the sequence is shifted, with respect to the position of the basic spot from which the sequence is derived, by a fraction of the interspot distance given by $\bar{g} \cdot \bar{R}$. The intensity of the spots further decreases with increasing value of $m$.

This formula can easily be generalised to two-dimensional interface modulated structures. Let the interface distances be $d_{1}$ and $d_{2}$ respectively with unit normals $\bar{n}_{1}$ and $\bar{n}_{2}$ and $\bar{R}_{1}$ and $\bar{R}_{2}$ as displacement vectors. The reciprocal lattice vectors of the superstructures are then given by

$$
\bar{H}=\bar{g}+1 / d_{1}\left(m_{1}-\bar{g} \cdot \bar{R}_{1}\right) \bar{n}_{1}+1 / d_{2}\left(m_{2}-\bar{g} \cdot \bar{R}_{2}\right) \bar{n}_{2},
$$

where $m_{1}$ and $m_{2}$ are integers. Formula (2) shows that the superstructure can be completely characterised by using information derived from the geometry of the diffraction pattern once the basic structure is known. By considering the variation of the intensity in the linear sequences it is in most cases possible to locate the basic spots and hence to identify the basic structure. The interspot distance within the sequences determines the distance $d$ between the interfaces, which furthermore are perpendicular
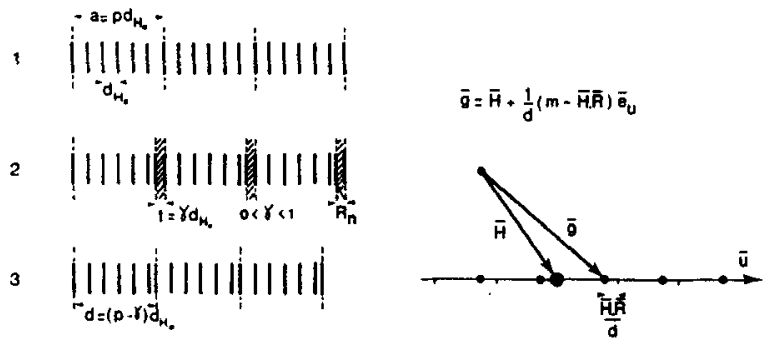

Figure 1. One-dimensional model of interface modulated structure and the associated diffraction pattern. 1. Basic structure with period $d_{H_{0}}$. 2. A slice of thickness $\gamma d_{H_{0}}$ is periodically removed. 3. Resulting modulated structure with period $d=(p-\gamma) d_{H_{0}}$. The diffraction vectors of the basic structure are represented by $\bar{H}$ and those of the modulated structure by $\bar{g}$. 
to the direction of the sequence. The fractional shifts, with respect to the positions of the basic spots, determine the projection of $\bar{R}$ along the considered zone. Two zones are required to determine $\bar{R}$ completely but in many cases the projection is sufficient. We shall discuss examples of the determination of one and two-dimensional superstructures using this method.

If different interface spacings occur in a strictly periodic succession a new period results and is thus revealed in the diffraction pattern. However, if the spacing between interfaces varies discontinuously in a relatively narrow interval, i.e. if a mixture of two or three different spacings occurs in an aperiodic manner the diffraction pattern will reveal the average spacing (Fujiwara 1957). It is then possible that arrays of superlattice spots associated with different basic spots do not match where they meet and give rise to what is sometimes called a "spacing anomaly".

It is furthermore possible that the modulating interfaces are systematically ledged or jogged in such a way that their average orientation encloses a small angle with a simple direction of the basic structure (Van Tendeloo and Amelinckx 1977). This situation gives rise in the diffraction pattern to what is sometimes called an "orientation anomaly". The arrays of superlattice spots associated with the different basic reflections are then no longer parallel with a simple direction of the basic reciprocal lattice, but enclose a small angle with it. Diffraction patterns containing spacing and (or) orientation anomalies are sometimes called pseudo-incommensurate in contrast with the truly incommensurate patterns observed in deformation-modulated transition metal dichalcogenides (Van Landuyt et al 1974a,b, 1978; Williams et al 1974; Wilson et al 1974, 1975; Scruby et al 1975; Williams 1976) and related compounds (Van Tendeloo et al 1977; Cornellisen et al 1978).

\section{Imaging in ordered alloys}

\subsection{General considerations}

The structure of a binary alloy, derived from a known basic structure, is established once the positions of the minority atoms are known. The minority atoms determine in fact the unit cell size. The superlattice reflections can be thought of as resulting from scattering by the arrangement of the minority atoms only, provided the latter are attributed a scattering amplitude equal to the difference of the scattering amplitudes due to the minority and majority atoms respectively. This difference is positive if the minority atoms are also the lighter atoms, otherwise it is negative. Using superlattice reflections only for image formation thus reveals the positions of the minority atoms, which may be sufficient for a number of purposes. If an image of the basic structure is also needed, we must include the basic reflections in the set of image-forming beams.

These considerations have led us to the use of several imaging methods, depending on the objective pursued and on whether or not two-dimensional images are required.

\subsection{One-dimensional images}

If only a one-dimensional representation of the structure is required, for instance because the structure is a long period one-dimensional superstructure, one can use the following imaging techniques: (figure 2) 


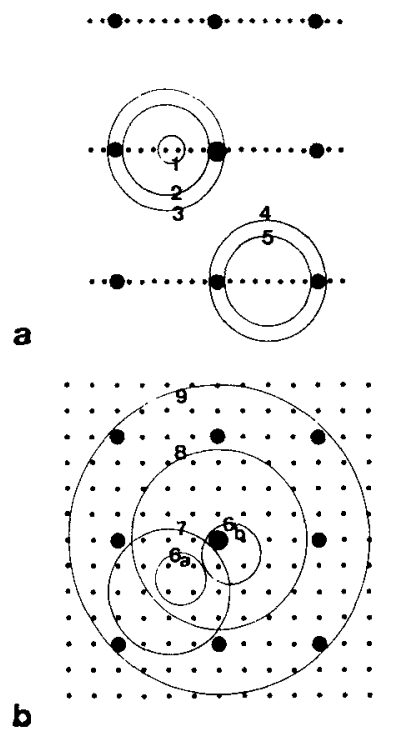

Figure 2. Schematic representation of different modes of beam selection referred to in the text. a. for onedimensional long period structures; the second row is the "central" row. b. for two-dimensional long period structures; the centre of the pattern corresponds with the incident beam.

Mode 1: One can select two neighbouring superstructure reflections belonging to the same basic spot in a row of spots passing through the origin, i.e. in a central row. One uses only one Fourier component and consequently the image, only reveals the long spacing (Menter 1956).

Mode 2: If all superstructure reflections belonging to a central row are used, excluding the basic reflections in the row, one obtains the distribution of long spacings. (Van Landuyt et al 1974).

Mode 5: One can also use a sequence of superlattice reflections from a non-central row. This is a useful mode if one wants to reveal polysynthetic sub-unit cell twinning (Van Landuyt et al 1974).

Mode 3: If one selects basic reflections as well as superlattice reflections in a central row (3) or a non-central row (5) one images also the set of lattice planes of the basic structure which is parallel with the periodic interfaces that produce the superstructure. Any variability of the long spacing is now imaged in terms of the spacing of the basic lattice.

If orientation and spacing anomalies occur in the diffraction pattern it is possible to produce images by collecting a pair of spots belonging to arrays associated with different basic reflections (De Ridder et al 1975, 1976). The so-formed images have a moiré like aspect; neither their direction nor their spacing is constant.

\subsection{Two-dimensional images}

Mode 6: If only the superlattice needs to be imaged it is sufficient to include only pairs of neighbouring superlattice spots in two directions. This can be done either in the dark field (6a) or in the bright field (6b). In the latter case the image is formed by the direct beam and the first shell of superlattice reflections around it.

Mode 7: All superlattice reflections are selected which are present within one mesh of the reciprocal lattice of the basic structure, excluding the direct beam and the basic reflections. This is clearly a multiple beam dark field image. The Fourier components 
contributing to the image are in general just sufficient to locate the positions of the minority atoms (Van Tendeloo et al 1978).

Mode 8: Except for the direct beam all other basic reflections are excluded but as many shells of superlattice reflections as feasible are admitted (Van Tendeloo and Amelinckx 1978; Van Tendeloo et al 1980; Hiraga et al 1980). The number of Fourier components required to image the columns of minority atoms is now redundant; however such columns will therefore be imaged as sharper dots than in the previous mode 7 .

Mode 9: All beams originating from the basic as well as from the superstructure reflections are selected, provided they do not correspond to spacings which are smaller than the instrumental resolving power of the microscope (Iijima et al 1974; Iijima 1975). For most current instruments this means that up to the first or possibly up to the second shell of reflections, due to an FCC matrix, can usefully be included. In our subsequent discussion we shall refer to these different modes and discuss examples of applications.

\section{High resolution studies of basic alloy structures}

\subsection{General}

A number of simple binary alloy superstructures of the FCC structure have been imaged in high resolution electron microscopy (Van Tendeloo et al 1978, 1981; Den Broeder et al 1981; Van Tendeloo and Amelinckx 1979). The separation of minority atoms in binary alloys is in many cases at least of the order of $2 \AA$, a distance which can conveniently be resolved.

Along certain zones the structure consists of columns, which contain only one atomic species, i.e. minority and majority atoms occur in separate columns. The distance between columns of minority atoms is then relatively the largest and it determines the unit mesh of the reciprocal lattice of the superstructure along this zone. Such zones are the most informative ones; along these it is possible to image only the minority atom columns by collecting superlattice reflections in the contrast aperture (mode 8). We shall say that the structure is a "column structure" along such rows.

\subsection{The $\mathrm{Ni}_{4} \mathrm{Mo}$ structure (Van Tendeloo et al 1978)}

The alloy $\mathrm{Au}_{4} \mathrm{Mn}$ which is isomorphous with $\mathrm{Ni}_{4} \mathrm{Mo}$ (figure 3a) is a particularly suitable object for high resolution electron microscopy, using the dark field superlattice mode (mode 7). The reciprocal lattice as viewed along the c-direction, is shown in figure $4 \mathrm{a}$, for a single variant. The diffraction conditions can then be chosen in such a manner that the projection of the centre of Ewald's sphere coincides with the centre of the octagon of superstructure reflections produced by the two coaxial variants (mode 6). Under these conditions all beams contributing to the images of coaxial variants enclose the same angle with the optical axis of the microscope and thus aberration-induced phase differences cancel. Particularly well-defined images result under these conditions.

The four beams collected in each variant provide the minimum number of Fourier components that allow to generate the pattern of minority atoms, without any redundant information.

Figure $5 \mathrm{a}$ shows an example of the $\mathrm{Au}_{4} \mathrm{Mn}$ perfect structure observed in this mode (mode 7); along the [001] zone, the $\mathrm{Au}_{4} \mathrm{Mn}$ structure is a column structure. 

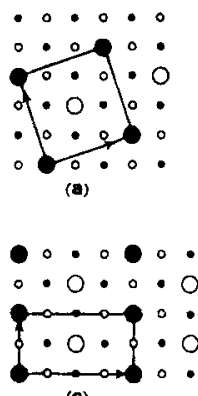

(c)

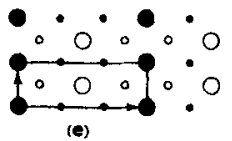

(e)

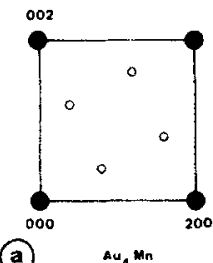

(a)

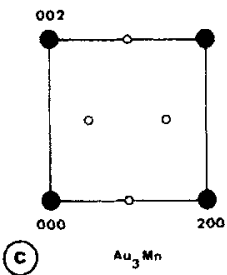

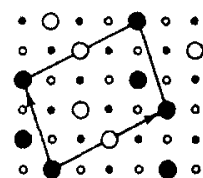

(b)

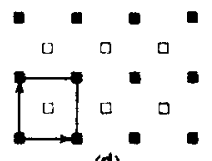

(d)
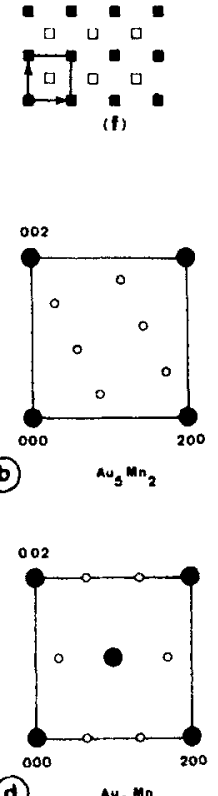

Figure 3. Schematic representation of different ordered structures derived from the FCC and the BCC structure. Large dots represent minority atoms. a. $\mathrm{Au}_{4} \mathbf{M n}$ structure viewed along the tetragonal c-axis. b. Monoclinic $\mathrm{Au}_{5} \mathrm{Mn}_{2}$ structure viewed along the twofold axis. $\mathrm{c}$. Tetragonal $\mathrm{DO}_{22}$ structure $\left(\mathrm{Au}_{3} \mathrm{Mn}\right)$ as viewed along the a-axis. d. The same structure viewed along the tetragonal c-axis. These three structures are derived from the $\mathrm{FCC}$ structure. e. Tetragonal $\mathrm{Cr}_{2} \mathrm{Al}$ structure viewed along the a-axis $\left(\mathrm{Au}_{2} \mathrm{Mn}\right.$ has the same structure). f. The same structure viewed along the $c$ axis. This structure is based on a BCC lattice.

Figure 4. Reciprocal lattice sections of the structures represented in figure 3 : In all cases a cube zone is shown. The full dots represent basic reflections, the open dots superstructure refiections.

We have also used the bright field basic lattice mode (mode 9). We now collect all beams within four unit meshes of the basic FCC reciprocal lattice, as well as the basic reflections themselves. The image now clearly contains information on the basic FCC structure, as well as on the superstructure. An image of a wedge shaped crystal obtained using this mode, is reproduced in figure $5 b$. It is clearly seen that in the thin part of the wedge the basic FCC structure is revealed. In the thicker parts, on the other hand, striking white dots reveal the positions of manganese columns. It is then possible to verify directly that the manganese columns occupy the positions with respect to the FCC lattice represented in the model of figure $3 \mathrm{a}$. The interpretation of the bright dots as marking the positions of the manganese columns is justified on the basis of computersimulated images, using the method described in $\$ 4.6$.

\subsection{The $\mathrm{DO}_{22}$ structure (Van Tendeloo et al 1983)}

A model of the $\mathrm{DO}_{22}$ structure is represented in figure 3. Figure $3 \mathrm{c}$ and figure $3 \mathrm{~d}$ are views along the $a$-axis and the $c$-axis respectively. It is clearly a tetragonal superstruc- 
ture of the FCC superstructure with an ideal $c / a$ ratio of 2 , its ideal composition is $\mathbf{A}_{3} \mathbf{B}$. The reciprocal lattice as viewed along the $a$-direction is represented in figure $4 \mathrm{c}$.

In specimens with nominal composition $\mathrm{Au}_{3} \mathrm{Mn}$, extensive regions of $\mathrm{DO}_{22}$ structure were obtained, as judged from the diffraction pattern. Images were obtained using the imaging mode 8 . A view along the a-direction is reproduced in figure $5 e$ whereas an image taken along the $c$-direction is shown in figure 5 f. From the two projections, the structure can be determined unambiguously.

Like $\mathrm{Au}_{4} \mathrm{Mn}$ viewed along the $c$-direction, the $\mathrm{DO}_{22}$ structure is a column structure when viewed along the $a$-direction, but not along the $c$-direction. The bright dots in figure $5 \mathrm{e}$ have the geometry and scale of the configuration of manganese columns in figure $3 \mathrm{c}$ as viewed along the $a$-zone. Along this zone there are in fact two types of manganese columns which differ in level by $a / 2$; they are imaged in the same way however, suggesting that the projected charge density is to a good approximation all that matters in determining the image. The gold columns are not imaged at all.

Along the $c$-direction on the other hand the structure is not a column structure; all manganese containing columns are mixed and have the composition AuMn. Apart from a relative shift of $c / 2$ in the $c$-direction they are all identical. One finds in fact that all such columns are imaged in the same way; the observed configuration of bright dots in figure $5 \mathrm{f} \mathrm{can}$ be compared with the scheme of figure $3 \mathrm{~d}$. There is apparently very little doubt left that under the conditions used, the manganese columns in the $\mathrm{DO}_{22}$ structure have been imaged.

\subsection{The $A u_{5} M n_{2}$ structure (Van Tendeloo et al 1978; Van Tendeloo and Amelinckx 1979)}

The $\mathrm{Au}_{5} \mathrm{Mn}_{2}$ structure is a monoclinic superstructure of the FCC structure; it is represented in projection of figure $3 \mathrm{~b}$; whereas the $(010)$ section of the reciprocal lattice is shown in figure $4 \mathrm{~b}$.

When viewed along the two-fold axis it is a column structure; the configuration of manganese columns is in fact shown in figure $3 \mathrm{~b}$. The distance between the components of a pair is only $0.25 \mathrm{~nm}$. In our most recent instrument we have been able to image the two columns in a pair as clearly separated bright dots (figure $5 d$ ) whereas in an earlier instrument we have only obtained elongated bright dots imaging a column pair (figure 5c).

The $\mathrm{Au}_{5} \mathrm{Mn}_{2}$ structure can in fact be described as an interface-modulated superstructure of the $\mathrm{DO}_{22}$ structure. When introducing periodic $A P B$ 's on (101) planes, with a displacement vector $1 / 4[201]_{\mathrm{DO}_{22}}$, and with a period of $\sim 0.62 \mathrm{~nm}$, one obtains the $\mathrm{Au}_{5} \mathrm{Mn}_{2}$ structure.

We shall discuss in $\S 6.2$ other related interface-modulated superstructures of the $\mathrm{DO}_{22}$ structure.

\subsection{The $\mathrm{Cr}_{2} \mathrm{Al}$ structure (Van Tendeloo et al 1981; Den Broeder et al 1981)}

The $\mathrm{Cr}_{2} \mathrm{Al}$ structure is based on a BCC lattice. It is a tetragonal superstructure with an ideal $c / a$ ratio of 3 ; its unit cell contains three всc unit cells (figure 3e). The ideal composition is clearly $A_{2} B$. Also $\mathrm{Au}_{2} \mathrm{Mn}$ has a similar structure. Views along the $a$-and $c$-directions are shown in figure $5 \mathrm{~g}, \mathrm{~h}$. The structure is a column structure along the [100], [010] and [111] zones, and obviously not along the [311] or [001] directions.

We have obtained clearly-defined structure images of $\mathrm{Cr}_{2} \mathrm{Al}$. viewed along the [100] 
direction (figure $5 \mathrm{~g}$ ). The $c / a$ ratio deduced from the image is about 2.9 in fair agreement with that deduced from x-ray diffraction. Along the $c$-axis all columns are identical and have the macroscopic composition $A_{2} B$. The reciprocal lattice, as viewed along the $a$-direction, is shown in figure $4 \mathrm{~d}$.

\subsection{Image simulation (Spence 1981; Van Dyck 1978)}

It is worth noting that in this paper we have intentionally and systematically used images which allowed a direct interpretation in terms of structure models. Such images can be obtained by making through focus series, but also more efficiently with an appropriately adapted microscope. Our instrument is equipped with an image intensifier system, making it possible to obtain the image on a television monitor at a magnification of $10^{7} \times$. Under these conditions it is possible to observe visually the bright dot pattern of minority atoms. This has made it possible to select systematically focusing conditions and thicknesses which produce this type of patterns, avoiding the need for through focus series.

In all cases so far studied, we could produce images such that the pattern of bright dots had the same scale and geometry as the arrangement of columns of minority atoms, strongly suggesting that we were in fact representing such columns as bright dots.

Moreover we have also shown that under the same imaging conditions, the coincidence columns of minority atoms along the inclined interface between two orientation variants are revealed as bright dots (Van Tendeloo and Amelinckx 1978). This can be considered as an experimental proof of our suggestion.

Along zones where all columns have the same but mixed composition, i.e. the macroscopic composition, all columns are represented by bright dots of the same intensity.

All these observations strongly suggest that the bright dots do represent columns of minority atoms along zones where minority and majority atoms occur in separate columns (so-called column structures). Nevertheless it is desirable to verify this interpretation by means of computed images, following a "trial and error" scheme. This is in any case required if we want to make sure that the bright dots occur at the sites of the minority atom columns and are not merely reproducing the correct configuration at a shifted position, however. It is perhaps an academic question if we want to obtain

Figure 5. High resolution images of different basic ordered structures all at the same magnification. In all cases the bright dots reveal the columns of minority atoms; their configurations can be compared with figure 3. The unit cell is outlined. a. $\mathrm{Au}_{\mathbf{4}} \mathrm{Mn}$ structure viewed along the tetragonal c-axis. $\mathbf{b}$. Wedge shaped crystal of the $\mathrm{Au}_{4} \mathrm{Mn}$ structure. In the thin part only the FCC structure $\left(a_{0} \sim 0.2 \mathrm{~nm}\right)$ is revealed; in the thicker parts the bright dots reveal the manganese columns. $c$. Monoclinic $\mathrm{Au}_{5} \mathrm{Mn}_{2}$ structure as viewed along the two-fold axis. The elongated dots represent close neighbour pairs of manganese columns. d. Monoclinic $\mathrm{Au}_{5} \mathrm{Mn}_{2}$ structure as viewed along the two-fold axis. The pairs of columns of manganese atoms are now revealed as separated dots. e. Tetragonal $\mathrm{Au}_{3} \mathrm{Mn}\left(\mathrm{DO}_{22}\right)$ structure $(c / a=2)$ as viewed along the a-axis. Only manganese columns are revealed. f. Tetragonal $\mathrm{Au}_{3} \mathrm{Mn}\left(\mathrm{DO}_{22}\right)$ structure as viewed along the c-axis. All columns are equivalent; they have a mixed composition. g. Tetragonal $\mathrm{Cr}_{2} \mathrm{Al}$ structure (also $\mathrm{Au}_{2} \mathrm{Mn}$ ) viewed along the a-axis $(c / a=3)$. In the thin part of the specimen the body-centered basic structure is revealed. Only minority atom columns are visible in the thicker part. $\mathbf{h}$. Tetragonal $\mathrm{Au}_{2} \mathrm{Mn}$ structure viewed along the c-axis. Faults in $(110)$ and $(1 \overrightarrow{10})$ are invariably present in $\mathrm{Au}_{2} \mathrm{Mn}$. 

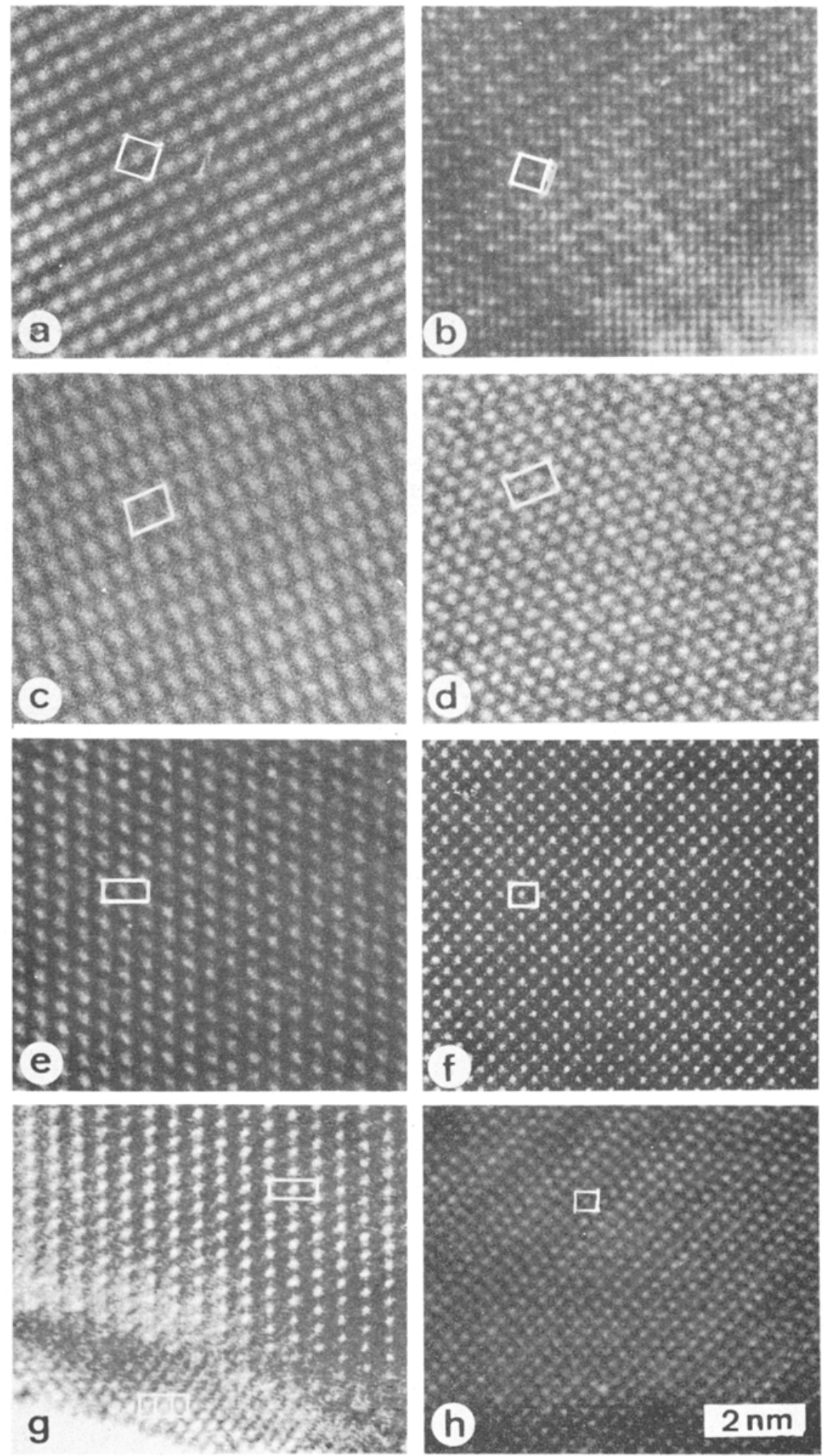
thickness variation

Au 4Mn
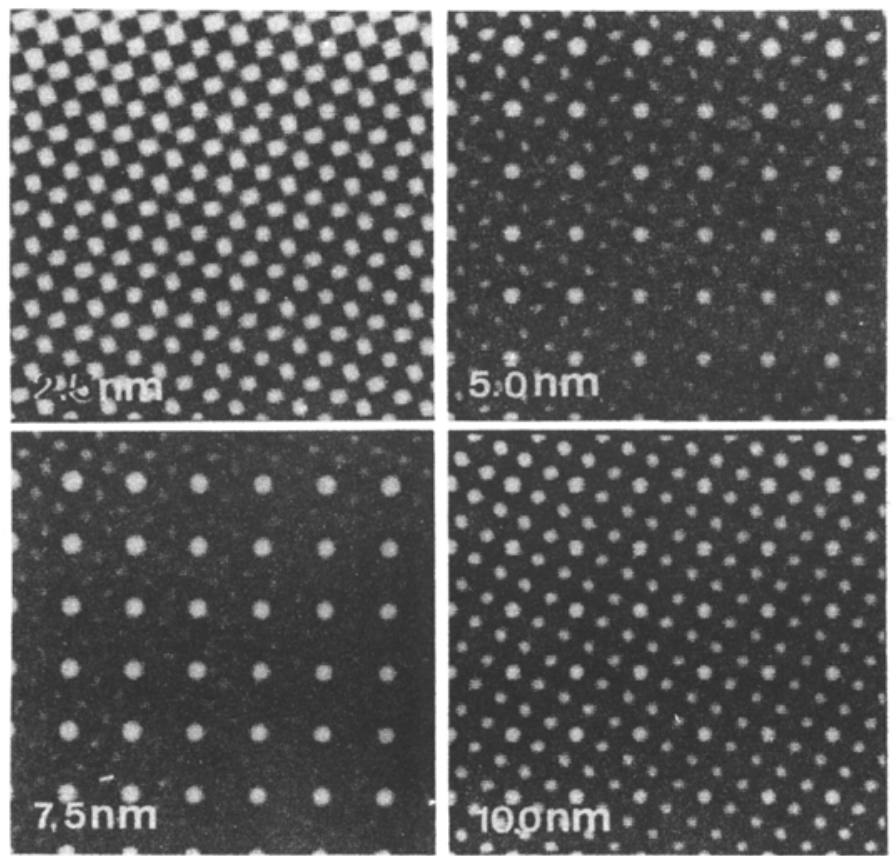

defocus:-100nm

foc.spread:5nm

Cs: $1.1 \mathrm{~mm}$

defocus variation
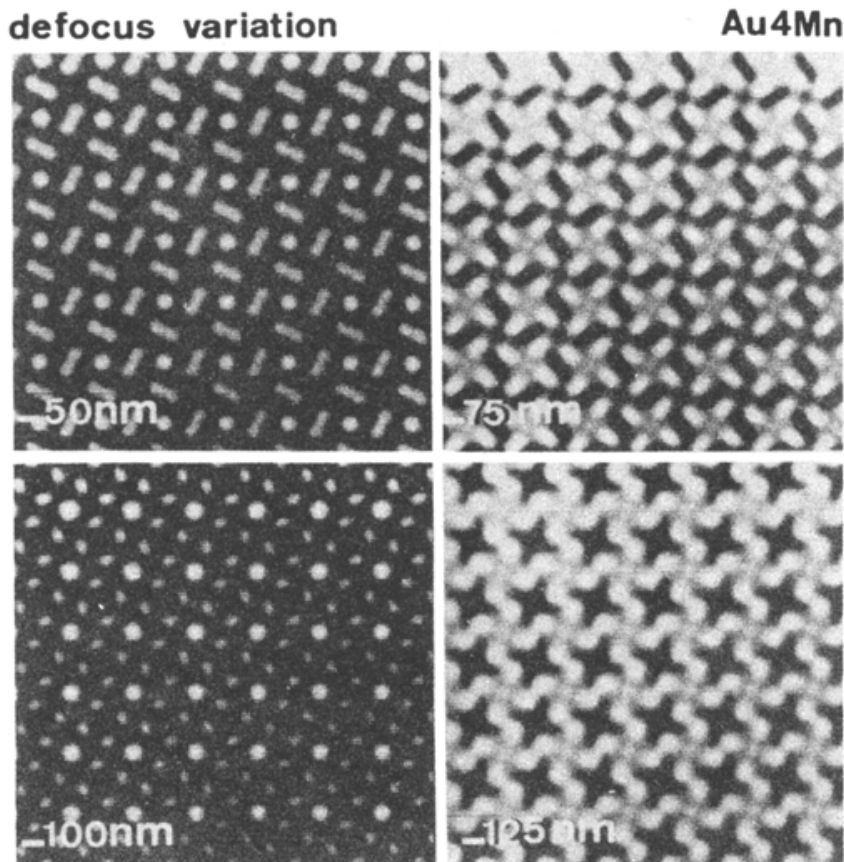

thickness: $5 \mathrm{~nm}$

foc.spread:10nm

Cs:1.1mm

Figure 6. Computer-generated images of $\mathrm{Au}_{4} \mathrm{Mn}\left(c_{s}=1.1 \mathrm{~mm}\right)$ (Courtesy Van Dyck). 

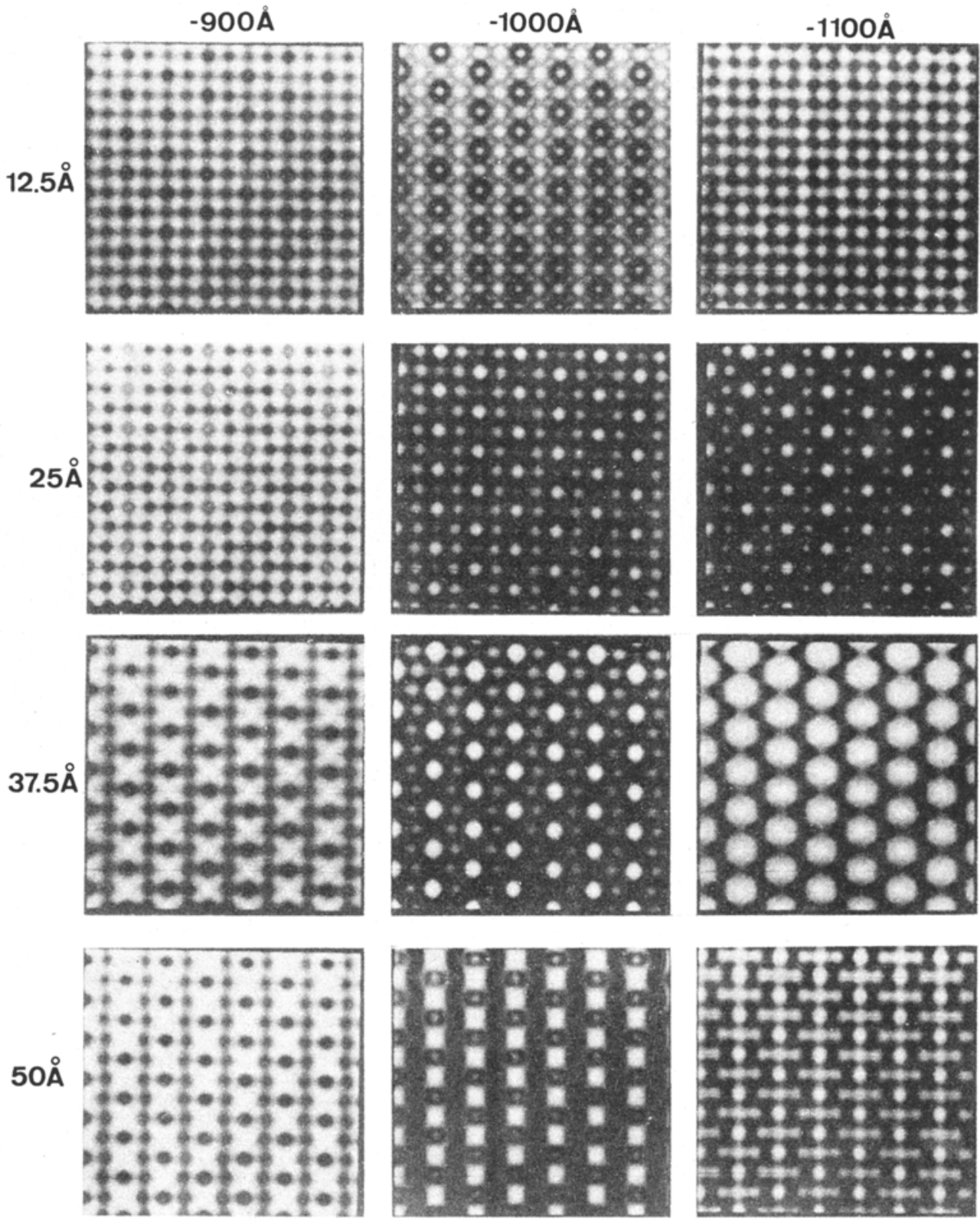

Cs: $1.1 \mathrm{~mm}$ div.:0 foc.spread:0

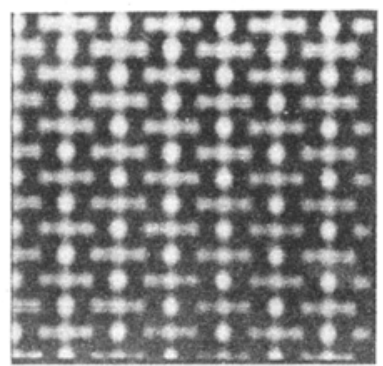

Au3Mn DO 22

Figure 7. Computer-generated images of the $\mathrm{Au}_{3} \mathrm{Mn}\left(\mathrm{DO}_{22}\right)$ structure.

information on the perfect structure or even on translation interfaces; this is no longer so if we study the interface between orientation variants or other defect structures such as dislocations.

In order to make this verification, it is necessary to produce computed images and compare these with the observations. The computation method used here is an 
improved version of the multislice method originally proposed by Cowley and Moodie (1957) and further developed by Van Dyck (1980). It fully takes into account multiple beam dynamical scattering in the crystal, as well as the phase shifts introduced by the aberration of the electron optical system and by defocussing $\left(c_{s}=1.1 \mathrm{~mm}\right.$ in our microscope). The final result of the calculation is plotted on a cathode ray screen and photographed, producing in this manner an image of the same nature as the electron micrograph.

We have computed a number of images of different basic structures, viewed along different directions, for a number of thicknesses and defocusvalues. We shall discuss here briefly two examples $\mathrm{Au}_{4} \mathrm{Mn}$ and $\mathrm{Au}_{3} \mathrm{Mn}\left(\mathrm{DO}_{22}\right.$ structure). The images were calculated on the assumption that the incident beam is parallel to the atom columns and that the superstructure spots as well as the first shell of basic spots are admitted through the objective aperture $(\bmod 9)$.

The sequence of computed images in figure 6 refers to $\mathrm{Au}_{4} \mathrm{Mn}$. For easy reference the left bottom corner of the frame was occupied in the model by a manganese column. The brightest dots clearly occur at the sites where manganese columns are located according to the model at least for a defocus value of -1000 to $-1200 \AA$ and a thickness in the range of $50 \AA$.

At small thicknesses the sites of the gold atoms are marked by weaker dots; at larger thicknesses the gold images become vanishingly weak (figure 6). This thickness behaviour is in agreement with the observation (figure $5 \mathrm{~b}$ ).

A similar sequence of computed images referring to the $\mathrm{DO}_{22}$ structure of $\mathrm{Au}_{3} \mathrm{Mn}$ is reproduced in figure 7. Again in this model a manganese column was placed in the left bottom corner of the frame. From the computed images we can conclude that in this case also the manganese columns are represented as bright dots in sufficiently thick foils. In thinner foils the gold atoms are also visible, as weaker dots however.

Qualitatively the contrast behaviour as a function of thickness can be understood by noting that the intensity of the superstructure beams builds up much more slowly as a function of thickness than the intensities of the beams due to the basic structure. Stated otherwise the depth variation of the intensities of the basic reflections is different from that of the superstructure reflections. At certain thicknesses the intensities of the basic beams may thus be weak whilst the superstructure beams may be relatively strong.

5. Domain structure in alloys (Van Tendeloo and Amelinckx 1974; Gaymont et al 1977)

\subsection{Orientation variants}

Long range ordering usually leads to a decrease in symmetry, the point group of the superstructure being a subgroup $H$ of the point group $G$ of the basic structure.

The superstructure can thus be formed in different orientations, related by symmetry operations of $G$, which were lost in the ordering process and which can thus no longer be present in $H$. Such orientation variants are a priori equally probable in a specimen formed in an isotropic environment.

The number of orientation variants is given by $n=$ order of $G /$ order of $H$. In many cases the set of operations relating the different orientation variants form a group by themselves; the variant generating point group $V$. If $V$ exists one can often choose different variant generating groups, which generate of course the same set of variants of 
the superstructure. The group $V$ has to be a subgroup of $G$, of order $n$, and which has no element in common with any of the different orientations of $H$, except for the unit element. Let for instance $G=\left(\frac{4}{m} \overline{3} \frac{2}{m}\right)($ order 48$)$ and $H=4 / m$ (order 8$)$ then one can choose as variant generating groups $V_{1}=32$ or $V_{2}=3 m$ both of order 6 .

The relation between $H$ and $V$ for a given group $G$ is reciprocal, i.e. $H$ is the variant generating group if the superstructure has the point group $V$.

The interface between different orientation variants will be called a "twin" boundary. An operation of $V$ relates the domains on either side of the interface. Two interfaces with separate pairs of domains of which the components are related by the same operation of $G$ will be considered as crystallographically equivalent. One pair of orientation variants can thus be brought into coincidence with the other pair by a symmetry operation of $G$. Crystallographically different interfaces will have associated operations of $V$ belonging to different classes of $G$. In the above mentioned example the operations of $V_{1}=32$ belong to two classes: (i) perpendicular twins: the two domains on either side of the interface are related by a rotation over $120^{\circ}$ about the three-fold axis along [111]; (ii) anti-parallel twins having the same four-fold axis (coaxial twins); the two domains are now related by a rotation of $180^{\circ}$ about a two-fold axis along a [110] direction perpendicular to [111]; in terms of the alternative variant generating group $V_{2}=3 \mathrm{~m}$ the second type of interface (anti parallel twins) separates domains related by a mirror passing through the threefold axis.

The $\mathrm{DO}_{22}$ structure occurs in three orientation variants represented schematically with respect to the basic FCC cube in figure $8 \mathrm{c}$. The $\mathrm{Au}_{4} \mathrm{Mn}$ structure can be formed in six orientation variants from any FCC matrix (figure $8 \mathrm{a}$ ). Finally the $\mathrm{Au}_{5} \mathrm{Mn}_{2}$ structure occurs in 12 orientation variants (figure 8b) based on the same FCC basic lattice (table 1).

\subsection{Translation variants}

During the ordering process also translation symmetry is lost, the Bravais lattice of the basic structure being a sublattice of the Bravais lattice of the superstructure, neglecting small deformations of the basic lattice resulting from ordering. A number of lattice vectors of the basic structure are no longer lattice vectors of the superstructure. All vectors describing such translations are obtained by connecting one lattice node of the

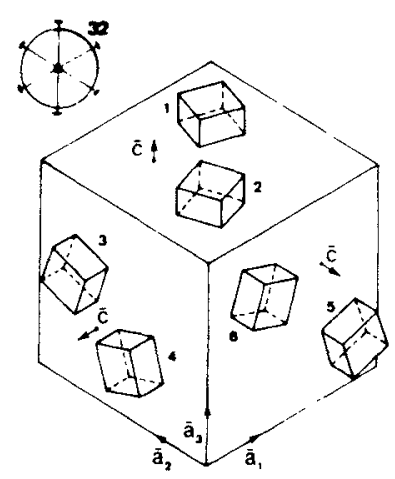

Figure 8a 
(b)

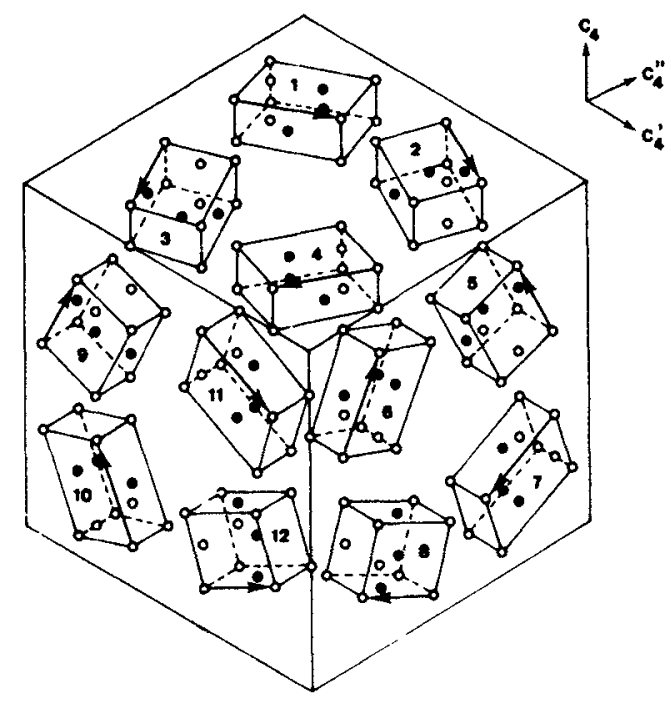

(c)

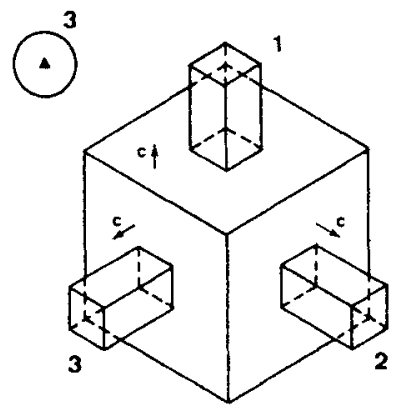

Figure 8. Unit cells of the orientation variants of three different ordered alloys represented with respect to the $\mathrm{FCC}$ cube. a. The 6 variants of the tetragonal $\mathrm{Au}_{4} \mathrm{Mn}_{\mathrm{n}}$ structure. The variant generating group is 32 with the three-fold axis along [111]. b. The 12 variants of the monoclinic $\mathrm{Au}_{5} \mathrm{Mn}_{2}$ structure. There is no variant generating group. $c$. The 3 variants of the tetragonal $\mathrm{DO}_{22}$ structure $(c / a=2)$. The variant generating group is 3 , with the three-fold axis along the [111] direction of the FCC structure.

superlattice with all nodes of the basic lattice contained within one primitive unit cell of the superlattice. The number of such translations $m$ is thus given by

$$
m=\frac{\text { volume of primitive unit cell of superstructure }}{\text { volume of primitive unit cell of basic structure }}
$$

The symmetry translations lost during the ordering process are possible displacement vectors for the interfaces separating translation variants. Such interfaces, called anti- 
phase boundaries, result because the superstructure can, with equal a priori probability, nucleate in different parallel positions related by these vectors.

Taking again the $\mathrm{Au}_{4} \mathrm{Mn}$ structure as an example one finds in this case $m=5$, i.e. there are four different types of anti-phase boundaries; they are represented in figure 9.1 .

In $\mathrm{Au}_{5} \mathrm{Mn}_{2}$ there are seven translation variants and hence six different types of anti phase boundaries (figure 9.2).

In the $\mathrm{DO}_{22}$ structure only four translation variants occur and hence three different APB's (figure 9.3).

The different geometrically allowed APB's need not all be actually present since in general their interfacial energies will be different. Moreover their energy also depends on the relative orientation of the displacement vector $\bar{R}$ and of the interface characterised by its unit normal $\bar{n}$.

Table 1. Different types of variants operating in ordered superstructures.

\begin{tabular}{|c|c|c|c|c|c|}
\hline Structure & $\begin{array}{c}G \\
\text { (order 48) }\end{array}$ & $H$ & $\begin{array}{c}\text { Orientation } \\
\text { variants } \\
n\end{array}$ & $\begin{array}{c}\text { Translation } \\
\text { variants } \\
m\end{array}$ & $\begin{array}{c}\text { Total number } \\
\text { of variants }\end{array}$ \\
\hline $\mathrm{Au}_{4} \mathrm{Mn}$ & $\frac{4}{m} \overline{3} \frac{2}{m}(\mathrm{FCC})$ & $4 / m$ & 6 & 5 & 30 \\
\hline $\mathrm{Au}_{3} \mathrm{Mn}\left(\mathrm{DO}_{22}\right)$ & $\frac{4}{m} \overline{3} \frac{2}{m}(\mathrm{FCC})$ & $4 / m 2 / m 2 / m$ & 3 & 4 & 12 \\
\hline $\mathrm{Au}_{5} \mathrm{Mn}_{2}$ & $\frac{4}{m} \overline{3} \frac{2}{m}(\mathrm{FCC})$ & $2 / m$ & 12 & 7 & 84 \\
\hline $\mathrm{Au}_{2} \mathrm{Mn}\left(\mathrm{Cr}_{2} \mathrm{Al}\right)$ & $\frac{4}{m} \overline{3} \frac{2}{m}(\mathrm{BCC})$ & $4 / m 2 / m 2 / m$ & 3 & 3 & 9 \\
\hline
\end{tabular}

1.

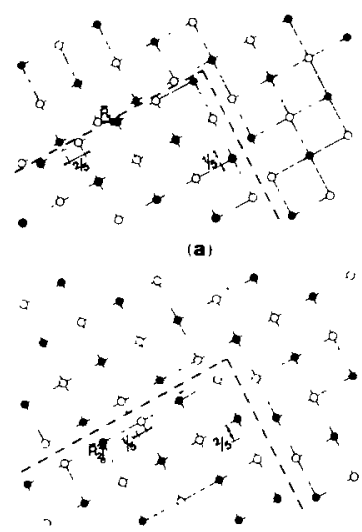

(c)

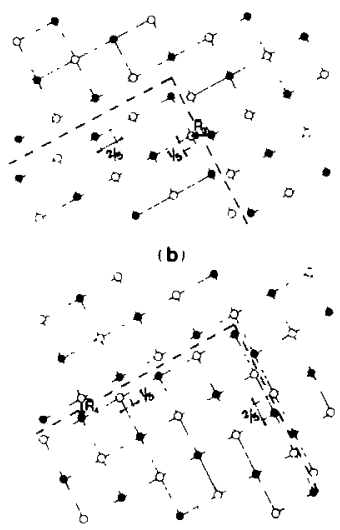

(d) 

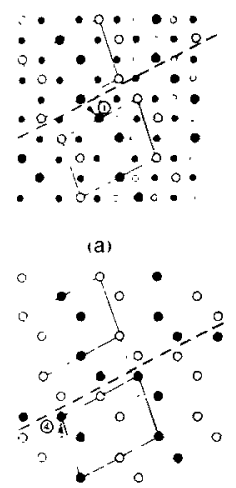

2. (d)

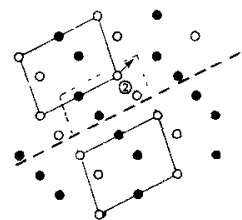

(b)

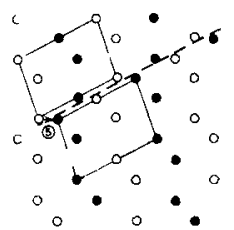

(e
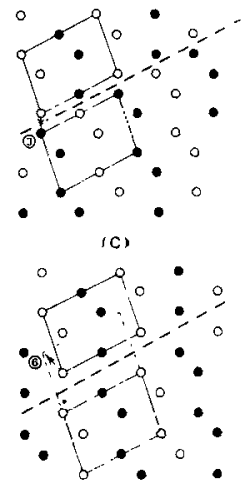

(f)
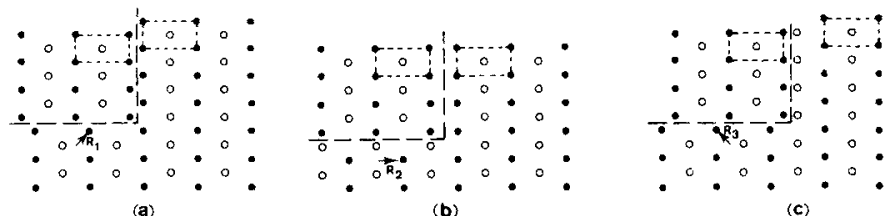

Figure 9. Outlines of the unit cells of different ordered structures illustrating the geometrically possible anti-phase boundaries. 1 . The $\mathrm{Au}_{4} \mathrm{Mn}$ structure occurs in 5 translation variants. Four different anti-phase boundaries are geometrically possible. 2. The $A_{u_{5}} \mathbf{M n}_{2}$ structure occurs in seven translation variants. Six different anti-phase boundaries are geometrically possible. 3. The $\mathrm{DO}_{22}$ structure can form in 4 different translation variants, leading to three different anti-phase boundaries.

\section{One-dimensional long-period superstructures}

\subsection{General}

For certain composition ranges and after suitable heat treatments a number of alloys adopt a one-dimensional long-period superstructure. Such structures have for instance been studied in the following alloy systems: CuAu (Scott 1960) Au-Zn (Schubert et al 1955; Iwasaki et al 1960; Iwasaki 1962; Van Tendeloo and Amelinckx 1977, 1978), Au-Mn (Van Tendeloo and Amelinckx 1978, 1981; Van Tendeloo et al 1978, 1979, 1980) AgMg (Fujiwara et al 1958; Schubert et al 1955). Cu-Sn (Schubert et al 1955; Van Sande et al 1978). We shall discuss three representative systems in order to illustrate the methods of interpretation and some characteristic features of such structures.

\subsection{The gold-manganese system}

In $\mathrm{Au}_{4-x} \mathrm{Mn}$ the one-dimensional long-period structure, derived from the $\mathrm{Au}_{4} \mathrm{Mn}$ structure, (figure 10b), was found (Van Tendeloo and Amelinckx 1981). The diffraction 
pattern is reproduced in figure $11 \mathrm{a}$, and represented schematically in figure $11 \mathrm{~b}$. From this scheme it is obvious that short sequences of equidistant superlattice spots along [110] directions are associated with the positions of the $\mathrm{Au}_{4} \mathrm{Mn}$ spots; the sequences are shifted with respect to such positions over fractions of the interspot distance, as indicated in table 2 . The displacement vector $\bar{R}=\frac{1}{10}[3 \overline{15}]$ is consistent with all fractional shifts as can easily be verified in table 2 by comparing calculated and observed values. The interspot distance leads to an interface spacing of $\sim 1 \cdot 1 \mathrm{~nm}$, the interfaces being parallel with the (110) planes. These data suggest the model represented in figure $12 \mathrm{a}$, when assuming that the white dots represent manganese columns. The structure is monoclinic with an ideal composition of $\mathrm{Au}_{33} \mathrm{Mn}_{9}$; it can be formed in twelve variants, four of which have their twofold axis parallel with the same cube direction. We call them co-axial variants. Each $\mathrm{Au}_{4} \mathrm{Mn}$ variant can give rise to two variants of the one-dimensional superstructure.

A number of one-dimensional long-period superstructures derived from the $\mathrm{DO}_{22}$ structure were found in alloys in the composition range $\mathrm{Au}_{3+x} \mathrm{Mn}_{\text {. }}$ (Van Tendeloo et al 1978; Wolf et al 1978; Van Tendeloo and Amelinckx 1983).
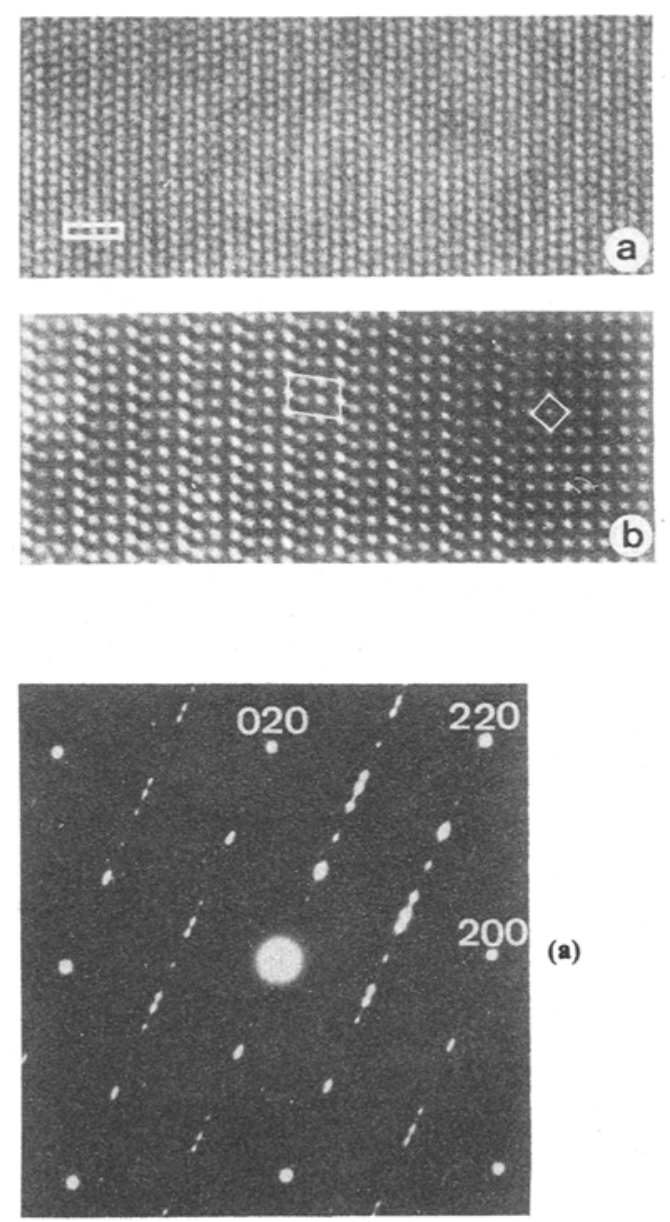

Figure 10. One-dimensional superstructures. a. Orthorhombic onedimensional superstructure in $\mathrm{Au}_{3} \mathrm{Zn}$. The stacking sequence is $2 \overline{2} 2 \overline{2}$. . The bright dots represent zinc columns. (courtesy Schrijvers). b. Monoclinic one-dimensional superstructure derived from the $\mathrm{Au}_{4} \mathrm{Mn}$ structure. In the right part of the micrograph a small area of $\mathrm{Au}_{4} \mathrm{Mn}$ structure is still present. The bright dots reveal manganese columns.

Figure 11 


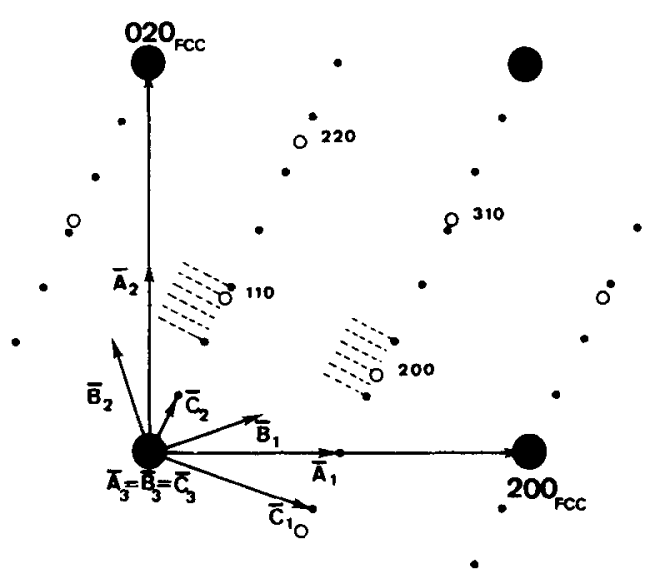

(b)

Figure 11. Diffraction pattern of the one-dimensional long-period superstructure of $\mathrm{Au}_{\mathbf{4}} \mathrm{Mn}$. a. observed pattern. b. schematic representation. The $\mathrm{Au}_{4} \mathrm{Mn}$ spots are represented as open dots.

Table 2. Fractional shift of the superlattice diffraction spots.

\begin{tabular}{lcc}
\hline & $\begin{array}{c}\text { Calculated } \\
\text { fractional shift } \\
\bar{R}=1 / 10[3 \overline{1} 5] \\
\bar{g} \cdot \bar{R}\end{array}$ & $\begin{array}{c}\text { Observed } \\
\text { fractional shift }\end{array}$ \\
\hline $\bar{g}\left(\mathrm{Au}_{4} \mathrm{Mn}\right)$ & $1 / 5$ & $1 / 5$ (or $4 / 5)$ \\
(1) 110 & $2 / 5$ & $2 / 5$ (or $3 / 5)$ \\
(2) 220 & $3 / 5$ & $3 / 5$ (or $2 / 5)$ \\
(3) 200 & $4 / 5$ & $4 / 5$ (or $1 / 5)$ \\
(4) 310 & 0 & 0 (or 1$)$ \\
(5) $3 \overline{1} 0$ & &
\end{tabular}
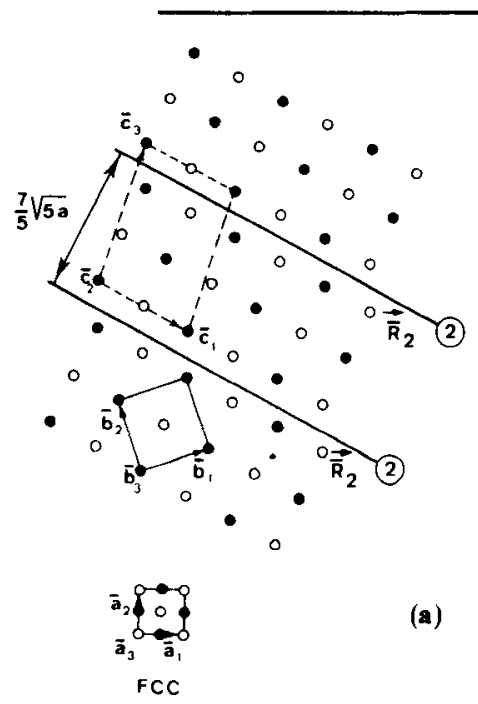

(a)

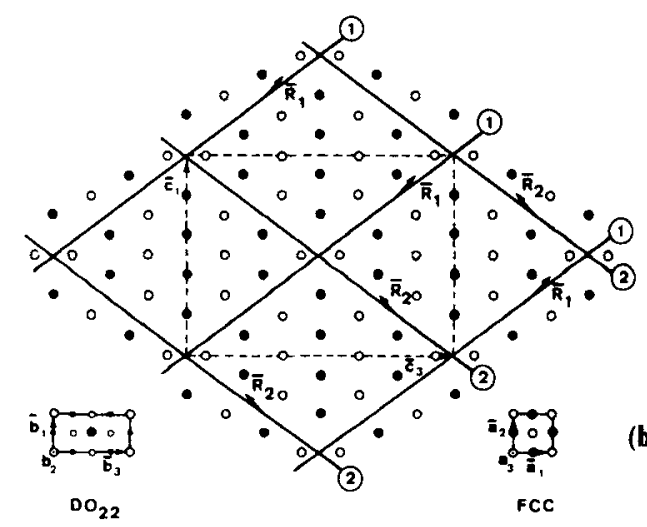

Figure 12. a. Model of the one-dimensional long-period superstructure of $\mathrm{Au}_{4} \mathrm{Mn}$ ( $c$. figure 10b). b. Lozenge-shaped island superstructure of the $\mathrm{DO}_{22}$ structure (cf. figure 27). 
An orthorhombic superstructure with a long period of about $20 \AA$, as observed at high resolution is shown in figure $13 \mathrm{a}$ together with its diffraction pattern. This superstructure results from the $\mathrm{DO}_{22}$ basic structure by the periodic introduction on
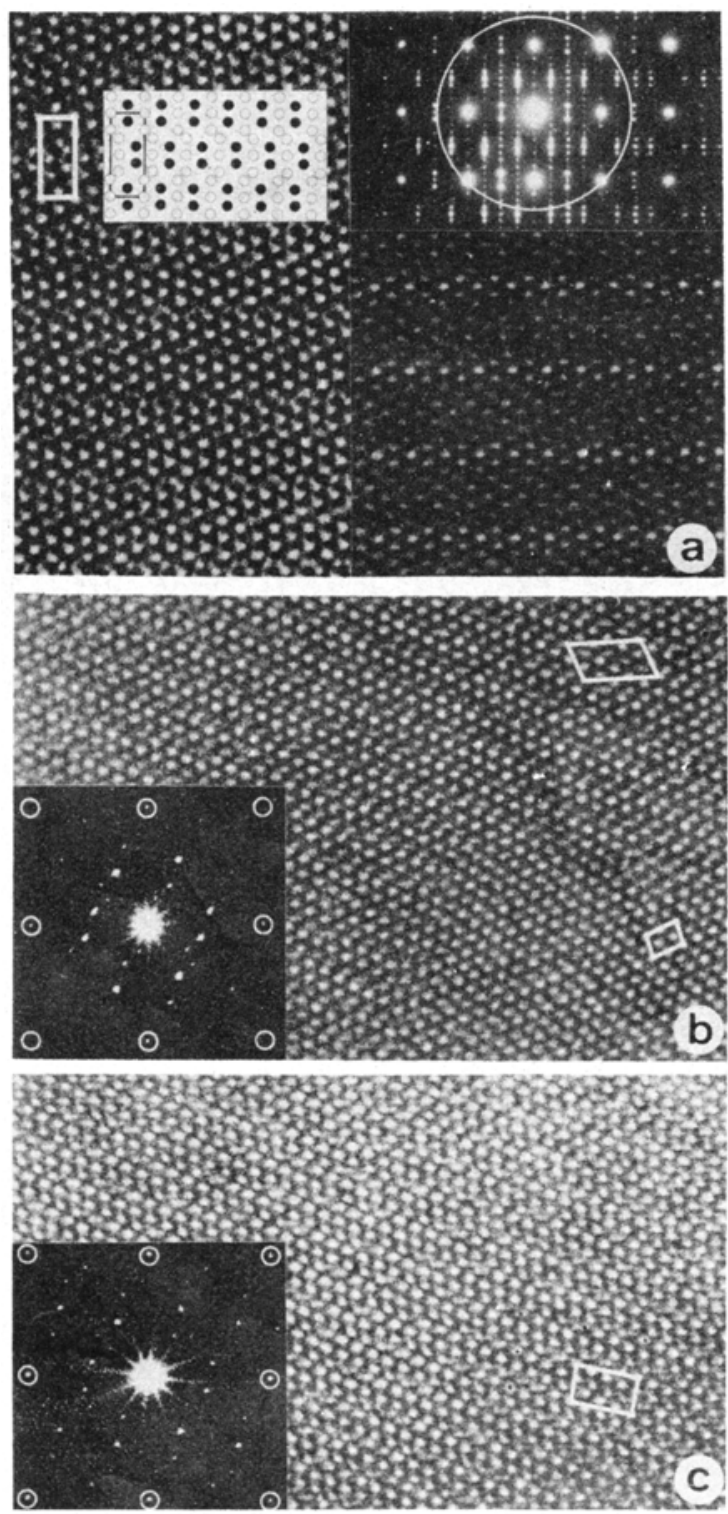

Figure 13. One-dimensional superstructures derived from the $\mathrm{DO}_{22}$ structure. a. Orthorhombic superstructure imaged under two different diffraction conditions; the right one emphasizing the $20 \AA$ spacing; the left one shows all atom columns with the same contrast. The insets show the diffraction pattern and the selected beams. Also the structure is represented in inset; only manganese columns are shown. b. Monoclinic long-period superstructure with anti-phase boundaries along (302) planes. The unit cell is outlined. The inset shows the optical diffraction pattern. In the right bottom part a region of $\mathrm{Au}_{5} \mathrm{Mn}_{2}$ structure is visible. $\mathbf{c}$. Monoclinic long period superstructure with anti-phase boundaries on (201) planes. The unit cell is outlined. The inset shows the optical diffraction pattern. 
(100) planes of APB's which have alternatively displacement vectors $1 / 4$ [201] and $1 / 4[201]$ and which have a $10 \AA$ spacing. All these structural features can be obtained from the geometry of the diffraction pattern as described in $\S 2$ and illustrated in the previous paragraph. A schematic model based on these considerations is reproduced in figure $14 \mathrm{a}, \mathrm{b}$; it can be compared with the high resolution image reproduced in figure 13a. Assuming the bright dots to represent the sites of manganese columns, excellent correspondence is obtained with the proposed model. The structure could also be considered as polysynthetically twinned. The anti-phase boundaries occurring in this structure are conservative; the ideal composition thus remains the same as that of the $\mathrm{DO}_{22}$ structure, i.e. $\mathrm{Au}_{3} \mathrm{Mn}$, unless the atomic positions along the APB 's are not all occupied by manganese in the manner assumed in the model. It is unlikely that in this case the formation of the superstructure is composition driven.

The orthorhombic structure is apparently generated from the $\mathrm{DO}_{22}$ structure by the propagation of hairpin shaped APB's. Figure 15 shows the interface between an area of $\mathrm{DO}_{22}$ structure and an area of long-period orthorhombic structure; hairpin shaped APB's are quite apparent. This configuration suggests that the atomic rearrangements required to generate the orthorhombic structure from the $\mathrm{DO}_{22}$ structure take place preferentially along the APB's and in particular at the tip of the hairpin.

For slightly different compositions we have found two monoclinic long period superstructures of the $\mathrm{DO}_{22}$ structure. The displacement vectors are now the same for all interfaces; they are again of the type $1 / 4$ [201] for both superstructures. However the orientations and spacings of the APB's are different for the two structures. In one case the APB's are in [302] planes and in the other in (201) planes (figure 13b,c) whereas the spacings are respectively $\sim 0.8 \mathrm{~nm}$ and $\sim 0.9 \mathrm{~nm}$. Models for both structures are represented in figures $16 \mathrm{a}, \mathrm{b}$, whereas figures $13 \mathrm{~b}, \mathrm{c}$ are the corresponding high resolution images. The imaging conditions were again chosen in such a way that the minority atoms are represented as bright dots. The correspondence between the models and the high resolution images is quite striking.

\subsection{The gold-zinc system}

We have studied the one-dimensional superstructures in the gold-zinc system at different levels of resolution. The average distance between anti-phase boundaries as deduced from the diffraction pattern varies between 2 and $2 \cdot 33$ FCC unit cells.

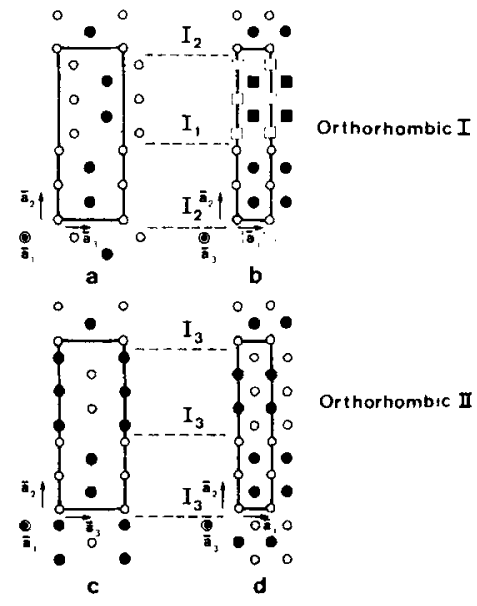

Figure 14. Models of two one-dimensional long-period superstructures of the $\mathrm{DO}_{22}$ structure. All anti-phase boundaries are conservative in the ideal structure. Figure 14a can be compared with figure 13a. The structure corresponding with figure $14 \mathrm{c}$ has been observed as well, but it is not reproduced here. 
Line resolution of the long period have demonstrated that this is due to the presence of a mixture of APB spacings equal to two and three FCC unit cells. In some cases the mixture is quite regular and structures, which can be represented schematically by means of the symbols $2 \overline{2} 3 \overline{2} 2 \overline{3}$ or $2 \overline{2} 2 \overline{3} 2 \overline{2} 2 \overline{3}$, may result (figure 17). (The number indicates the width of successive domain strips in terms of zinc columns; the minus sign indicates a displaced strip). After long annealing the spacing tends to become uniform; the structure symbol then becomes $2 \overline{2} 2 \overline{2}$. An atomic resolution picture of this structure is reproduced in figure $10 \mathrm{a}$. The antiphase boundaries are conservative and the ideal composition thus remains $\mathrm{Au}_{3} \mathrm{Zn}$.

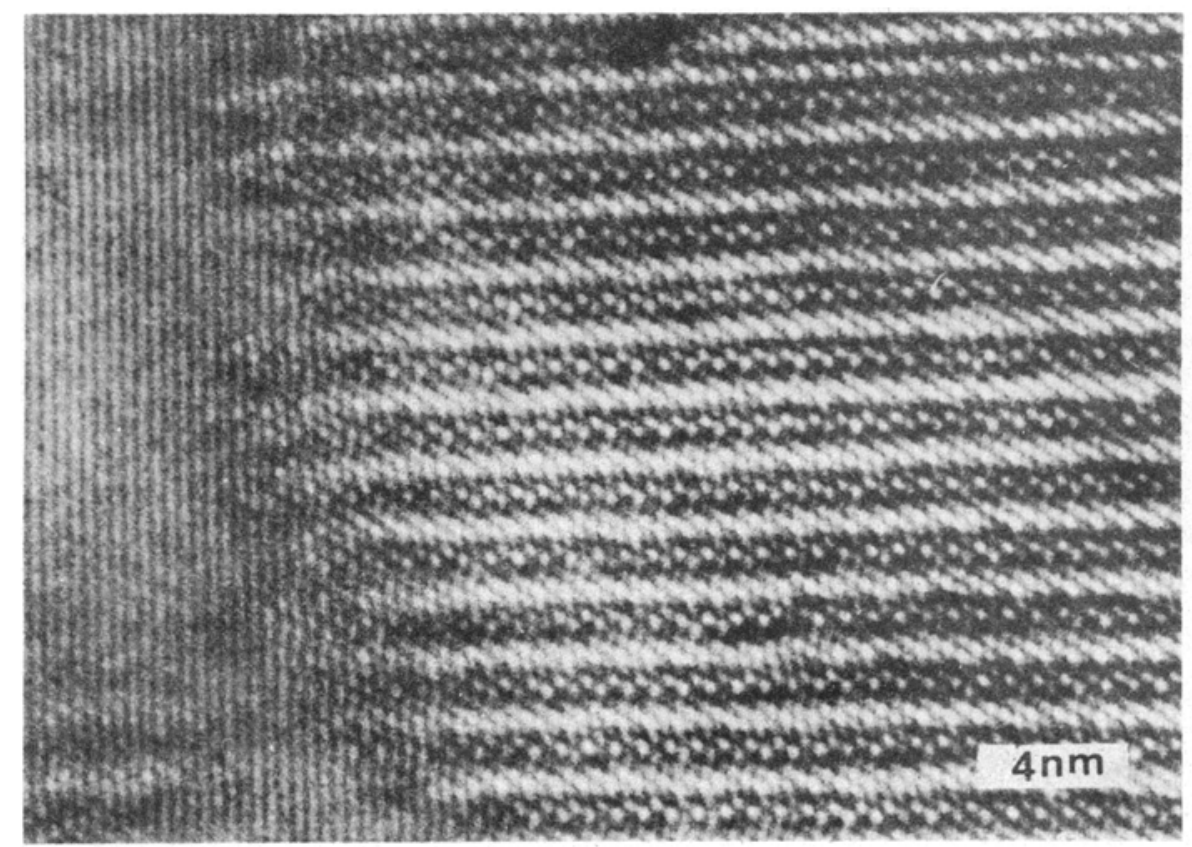

Figure 15. Interface between region of $\mathrm{DO}_{22}$ structure (left) and a region of orthorhombic I structure. The anti-phase boundaries propagate into the area of $\mathrm{DO}_{22}$ structure as hairpins.

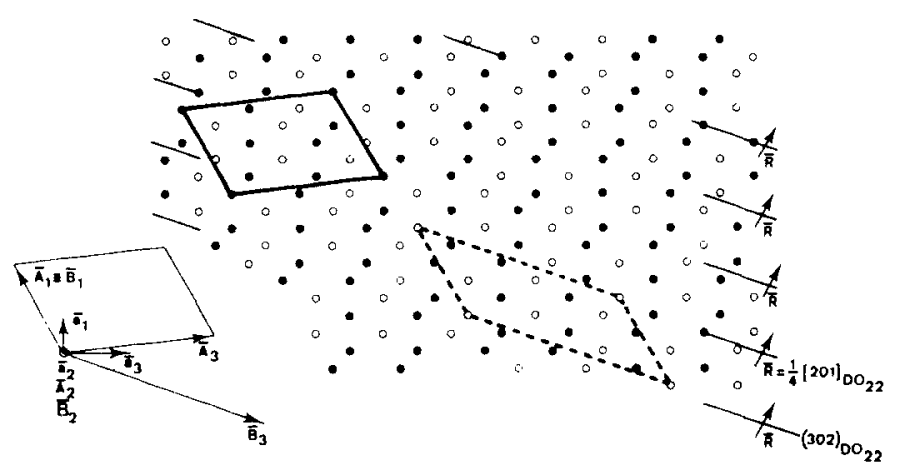

Figure 16a 
(b)

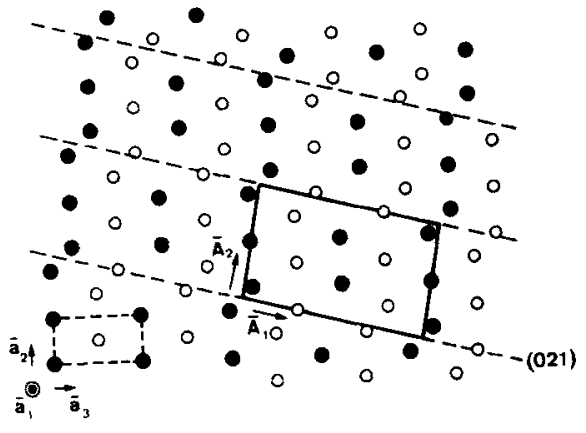

Figure 16. Models for monoclinic one-dimensional long-period superstructures of the $\mathrm{DO}_{22}$ structure. a. the APB's are on (302) planes. b. the APB's are on (201) planes. The displacement vector is the same in both cases.

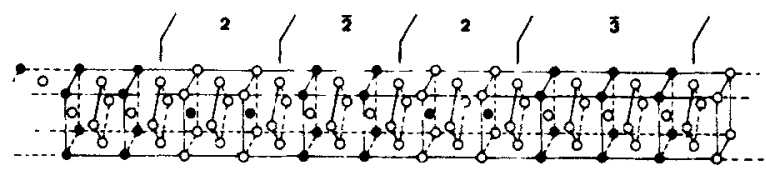

Figure 17. Model for the one-dimensional long-period superstructure in $\mathrm{Au}_{3+x} \mathrm{Zn}$.

After short annealing treatments at $250^{\circ} \mathrm{C}$ gold-zinc alloys with a composition of $\mathrm{Au}_{3+x} \mathrm{Zn}$ produce pseudo-incommensurate diffraction patterns, i.e. superstructure spot arrays associated with different basic spots do not match where they meet. Moreover the spot arrays often enclose a small angle with the cube direction. Lattice fringes reveal that the first effect is due to the presence of a mixture of spacings of 2 and 3 FCC unit cells wide; the diffraction pattern only reveals the average spacing. The second effect has to be attributed to systematic ledging of the anti-phase boundaries, causing the average orientation of the APB's to deviate slightly from the cube plane. This is represented schematically in figure 18. Presumably on further annealing the ledges migrate along the APB's producing in fact a lateral displacement of the APB's over a distance equal to the height of the ledge. This is possibly the mechanism by which, on annealing, the spacings become uniform. High resolution images show that the ledges do not have an abrupt structure, as suggested by the schematic of figure 18 , but have a rather more gradual shape (figure 19).

Peculiar static configurations of anti-phase boundaries have been interpreted in terms of the formation and (or) elimination mechanism of long period APB structures (Van Tendeloo and Amelinckx 1977, 1978)

\subsection{The alloy $\mathrm{Cu}_{3} \mathrm{Sn}$}

The structure of $\mathrm{Cu}_{3} \mathrm{Sn}$ is a periodic anti-phase boundary structure derived from an orthorhombic superstructure which is itself based on the hexagonally close packed structure (Schubert et al 1955). The lattice parameters of the long period superstructure $a_{s}, b_{s}$ and $c_{s}$ are given in terms of the lattice parameters of the basic orthorhombic structure by the relation

$$
a_{s}=2 a_{0}, b_{s}=8-10 b_{0}, c_{s}=c_{0} .
$$

The observations prove that the $b_{s}$ parameter may be varied by the addition of aliovalent impurities such as $\mathrm{Ni}$ and $\mathrm{Zn}$. 
The structure is represented in figure 20 ; it was assumed that $b_{s}=10 b_{0}$. The APB's are in $(010)$ planes and the displacement vector is $\bar{R}=1 / 2$ [100]. The observations on this alloy illustrate how the selection of an appropriate set of beams affects the final image and allows one to obtain the information best adapted to specific purposes. $\mathrm{A}$

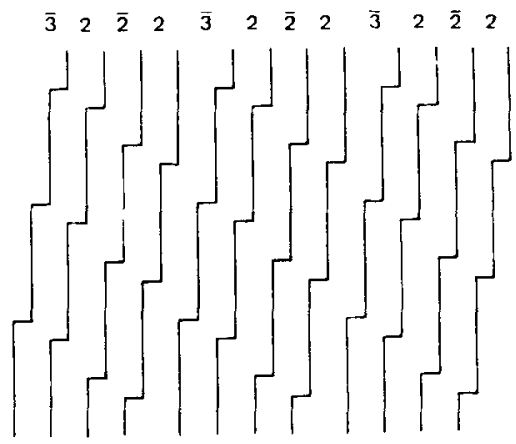

Figure 18. Systematic ledging of the antiphase boundaries leads to a change in their average orientation. The strips with a width of 3 units are distributed more uniformly after ledging than before.

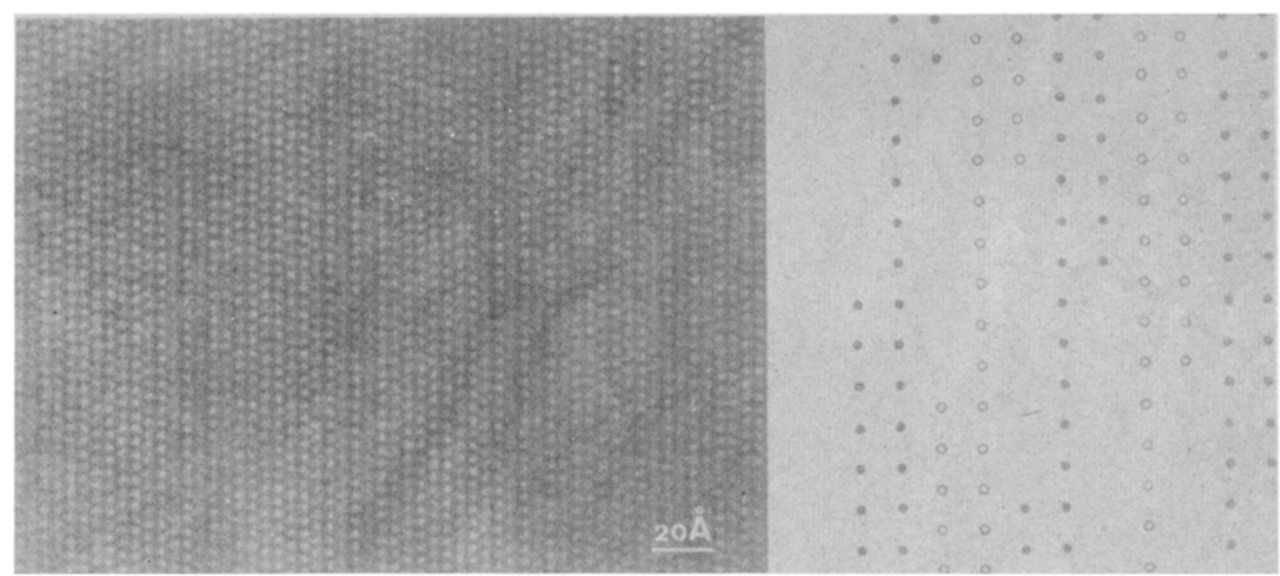

Figure 19. High resolution image of ledged $A P B$ 's in $\mathrm{Au}_{3+x} \mathrm{Zn}$. The real structure of the ledges is much more gradual than in the schematic representation of figure 18 (courtesy Schrijvers).
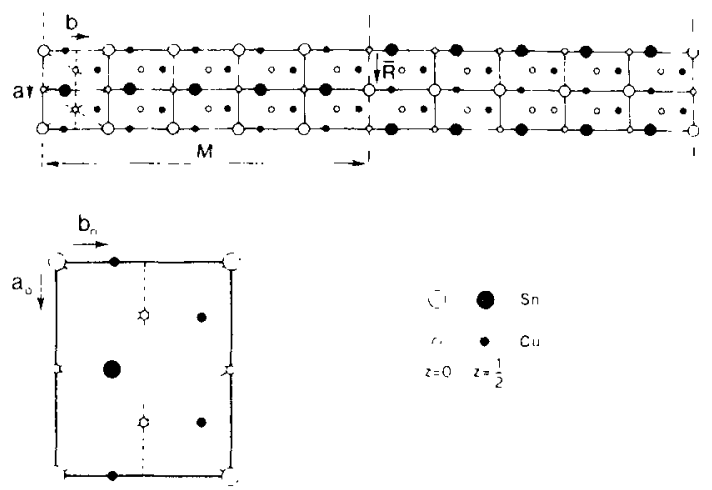

Figure 20. Model for the long period structure in $\mathrm{Cu}_{3} \mathrm{Sn}$. Also the basic structure derived from a HCP stacking is shown. 
typical diffraction pattern is reproduced in figure 21 and represented schematically in figure $21 \mathrm{~b}$, after omission of double diffraction spots (Van Sande et al 1978).

A rudimentary lattice image is obtained by collecting a sequence of satellite spots of the row $h=1$ in the objective aperture. Such dark field images only reveal the APB's as fine straight lines (figure 22a). Apart from occasional isolated faults the spacing is quite constant in well-annealed specimens; it corresponds to half the spacing deduced from a diffraction pattern; this is in accord with the model, since, the repeat distance contains two APB strips (figure 20). In nominally stoichiometric specimens the line spacing is $5 b_{0}$; we shall represent the long-period structure by the symbol $5 \overline{5}$, (the minus sign means displaced; the numbers indicate the width of the strips).

When collecting in the objective aperture the basic spots along the linear arrays of superlattice spots, lattice fringes due to the basic structure can be revealed as well.

It is then possible to observe directly the width of the domain strips in terms of unit cells of the basic structure (figure 22b).

When collecting beams belonging to at least two neighbouring arrays of satellite spots, as shown in the inset of figure $22 \mathrm{c}$, one obtains a two-dimensional representation of the structure such as the one reproduced in figure 22c. Darker bands now mark the

(a)

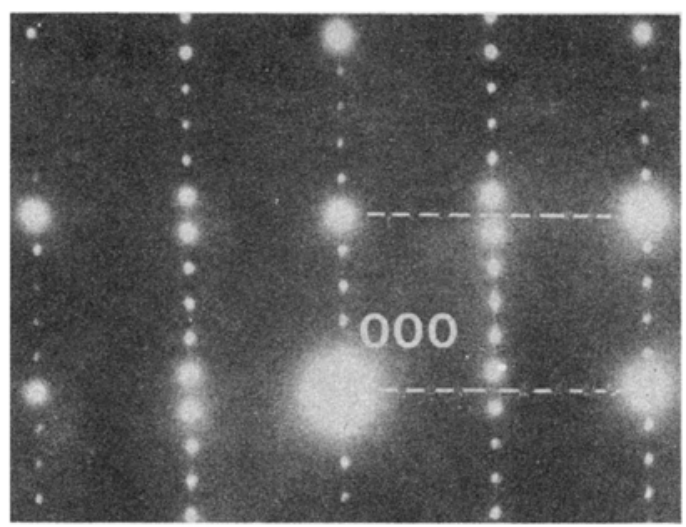

(b)

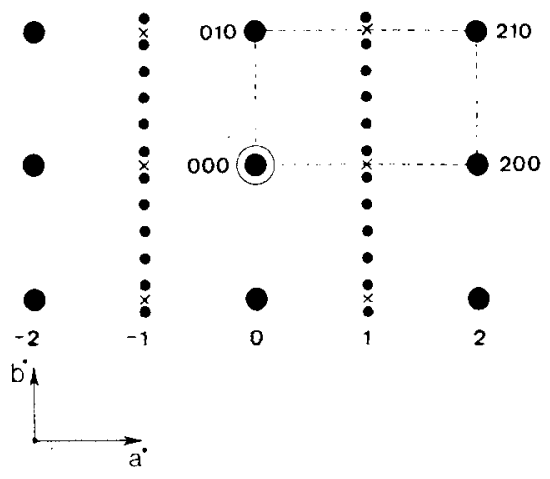

Figure 21. Electron diffraction pattern of $\mathrm{Cu}_{3} \mathrm{Sn}$. a. observed pattern. b. schematic representation. 


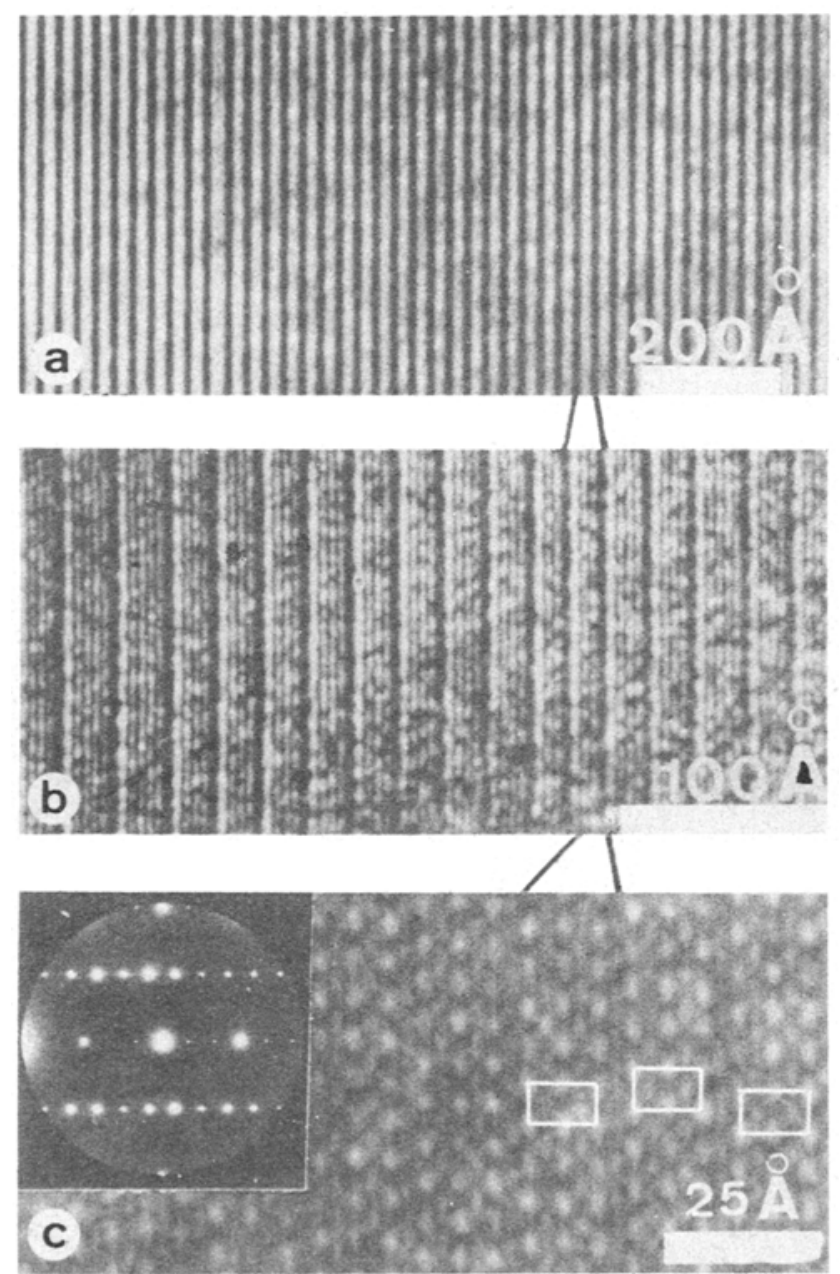

Figure 22. Images of the $\mathrm{Cu}_{3} \mathrm{Sn}$ structure at different levels of resolution. a. onedimensional image: the long spacing is revealed (mode 1). b. one-dimensional image: the spacing of the basic structure is revealed (mode 3). c. two-dimensional image (mode 8 ). The minority atoms are revealed as bright dots.

APB's. The scale and configuration of the white dots within the domains are the same as those of the minority atoms ( $\mathrm{Sn})$. The shift of the structure along the APB's is quite evident and in agreement with the model of figure 20.

The different levels of the resolution have been used for different purposes. The simple superlattice fringes have been used to study orientation variants; since the fringe orientation reveals directly the three possible directions of the $b$-directions with respect to the hexagonal basic structure. The lattice fringes of the basic structure have been used to study the changes in superperiod in alloys with addition of zinc and nickel; in particular the distribution of superlattice spacings becomes immediately evident. The diffraction pattern allows on the other hand to determine the average spacing. In quenched specimens the distribution is quite irregular. The "atomic" resolution image 
finally exhibits directly the configuration of atoms along APB's, and reveals the shift of the structure along them.

\section{Two-dimensional island structure}

\subsection{Derivatives of the $\mathrm{Cu}_{3} \mathrm{Au}$ structure}

Several types of two-dimensional long period superstructures have been described in the literature (Terasaki and Watanabe 1981). A number of them is derived from the $\mathrm{Cu}_{3} \mathrm{Au}$ ordered structure, which is based on an FCC lattice. The anti-phase boundaries are situated in mutually perpendicular cube planes giving rise to square or rectangular islands of $\mathrm{Cu}_{3} \mathrm{Au}$ structure. One family of APB's is usually conservative and the other one non-conservative; both have a displacement vector of the type $1 / 2[110]_{\mathrm{FCC}}$. A representative example of this type of structure is found in $\mathrm{Cu}_{3}$ in which the islands contain somewhere between $2 \times 3$ and $4 \times 6$ palladium columns, depending on the exact composition of the alloy. It is represented in figure 23 whereas an observed example is reproduced in figure 24 (Watanabe et al 1955; Schubert et al 1955).

Similar structures with different island sizes have been observed in $\mathrm{Au}_{3} \mathrm{Mg}$ (Airo 1978; Terasaki and Watanabe 1979), $\mathrm{Au}_{3} \mathrm{Zn}$ (Van Tendeloo and Amelinckx 1978a; Wilkens and Schubert 1957; Iwasaki et al 1960; Vanderschaeve 1980; Iwasaki 1962).

7.2 Derivatives of the $A u_{4} M n$ structure (Van Tendeloo et al 1980; Hiraga et al 1980; Van Tendeloo and Amelinckx 1981)

A quite different square island structure was found in alloys with composition $\mathrm{Au}_{4-x} \mathrm{Mn}$. It is derived from the $\mathrm{Au}_{4} \mathrm{Mn}$ structure (figure 25).

The antiphase boundaries with a displacement vector of the type $1 / 2[110]_{\mathrm{FCC}}$ are situated in mutually perpendicular planes, consisting of segments of $(110)$ and $(1 \overline{1} 0)$ planes, their average orientation enclosing a small angle with these planes. As yet only one such structure, with islands containing $3 \times 3$ manganese columns has been found (figure 26). Both families of anti-phase boundaries are non-conservative; the ideal composition of this phase is $\mathrm{Au}_{31} \mathrm{Mn}_{9}$. This structure is in fact the two-dimensional analogue of the one-dimensional structure described in $\S 6.2$.

\subsection{Derivatives of the $\mathrm{DO}_{22}$ structure}

A third type of two-dimensional long period structure derived from the $\mathrm{DO}_{22}$ structure consists of parallelogram or lozenge shaped islands containing $3 \times 3,3 \times 4$ and $4 \times 4$

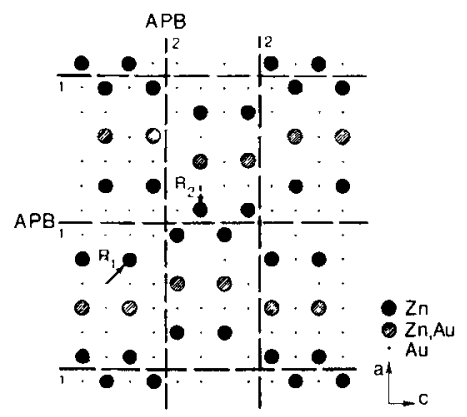

Figure 23. Schematic representation of the $\mathrm{Cu}_{3} \mathrm{Pd}$-type of two-dimensional long period superstructure; the structure shown refers actually to $\mathrm{Au}_{3+x} \mathrm{Zn}$. The vertical APB's are conservative, whereas the horizontal ones are nonconservative. 


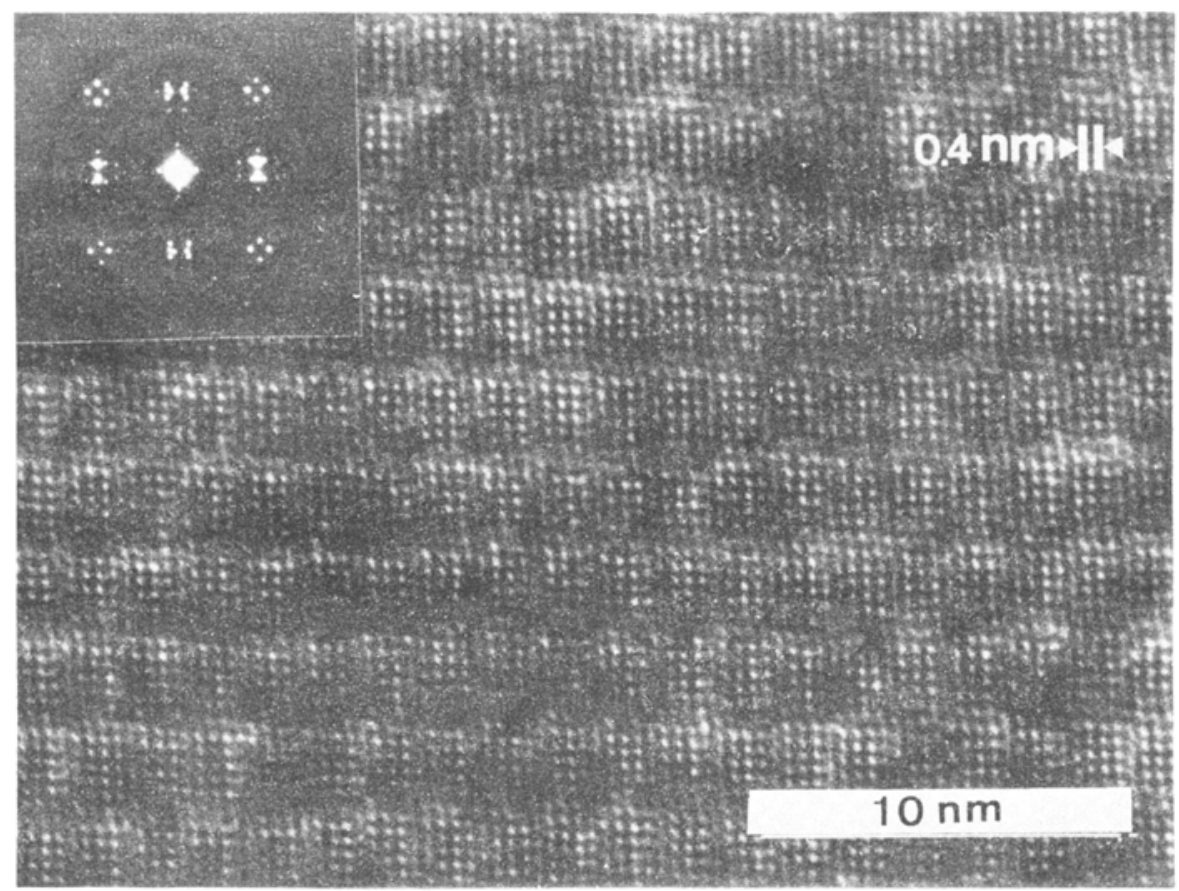

Figure 24. Orthorhombic $\mathrm{Cu}_{3} \mathrm{Pd}$ structure consisting of islands containing $4 \times 6$ columns of minority atoms. The inset shows the part of the diffraction pattern used for imaging.

manganese columns (figure 27a,b). The displacement vectors of the anti-phase boundaries are $1 / 4$ [201] and $1 / 4$ [201] . The islands are limited by segments of $\{101\}$ type planes; the average orientations of the anti-phase boundaries however deviate somewhat from these planes; they enclose an angle of $71^{\circ}-77^{\circ}$ (figure $12 \mathrm{~b}$ ). The ideal composition depends of course on the island size; for a $3 \times 3$ island size it is $\mathrm{Au}_{31} \mathrm{Mn}_{9}$.

In $\mathrm{Au}_{4-x} \mathrm{Mg}$ a monoclinic island structure with ideal composition $\mathrm{Au}_{15} \mathrm{Mg}_{4}$ was discovered (figure 28). It can be described as being derived either from the $\mathrm{DO}_{22}$ structure or from the $\mathrm{Cu}_{3} \mathrm{Au}$ structure. It consists of rows of square islands, limited by cube planes, containing $2 \times 2$ magnesium columns. The average orientations of the anti-phase boundaries are (201) and $\left(\begin{array}{lll}1 & 0 & 10\end{array}\right)$.

Recently a new type of periodic anti phase boundary structure, derived from the $\mathrm{DO}_{22}$ structure, was found in an alloy with nominal composition $A u_{77.5} \mathbf{M n}_{22.5}$. The non-conservative APB's are now on (001) planes and the displacement vector is $1 / 4$ [201]; the domain strips contain one $\mathrm{DO}_{22}$ unit cell. Its ideal composition is $\mathrm{Au}_{7} \mathrm{Mn}_{3}$. (Van Landuyt et al 1984).

\section{Observations of domain structures}

We shall now briefly discuss three examples of domain structures in the ordered alloys $\mathrm{Au}_{4} \mathrm{Mn}, \mathrm{Au}_{3} \mathrm{Mn}\left(\mathrm{DO}_{22}\right)$ and $\mathrm{Au}_{5} \mathrm{Mn}_{2}$ (figure 29). In the two coaxial variants of the $\mathrm{Au}_{4} \mathrm{Mn}$ structure all manganese columns are parallel with the common four-fold axis. It is therefore possible to image the two orientation variants simultaneously. 


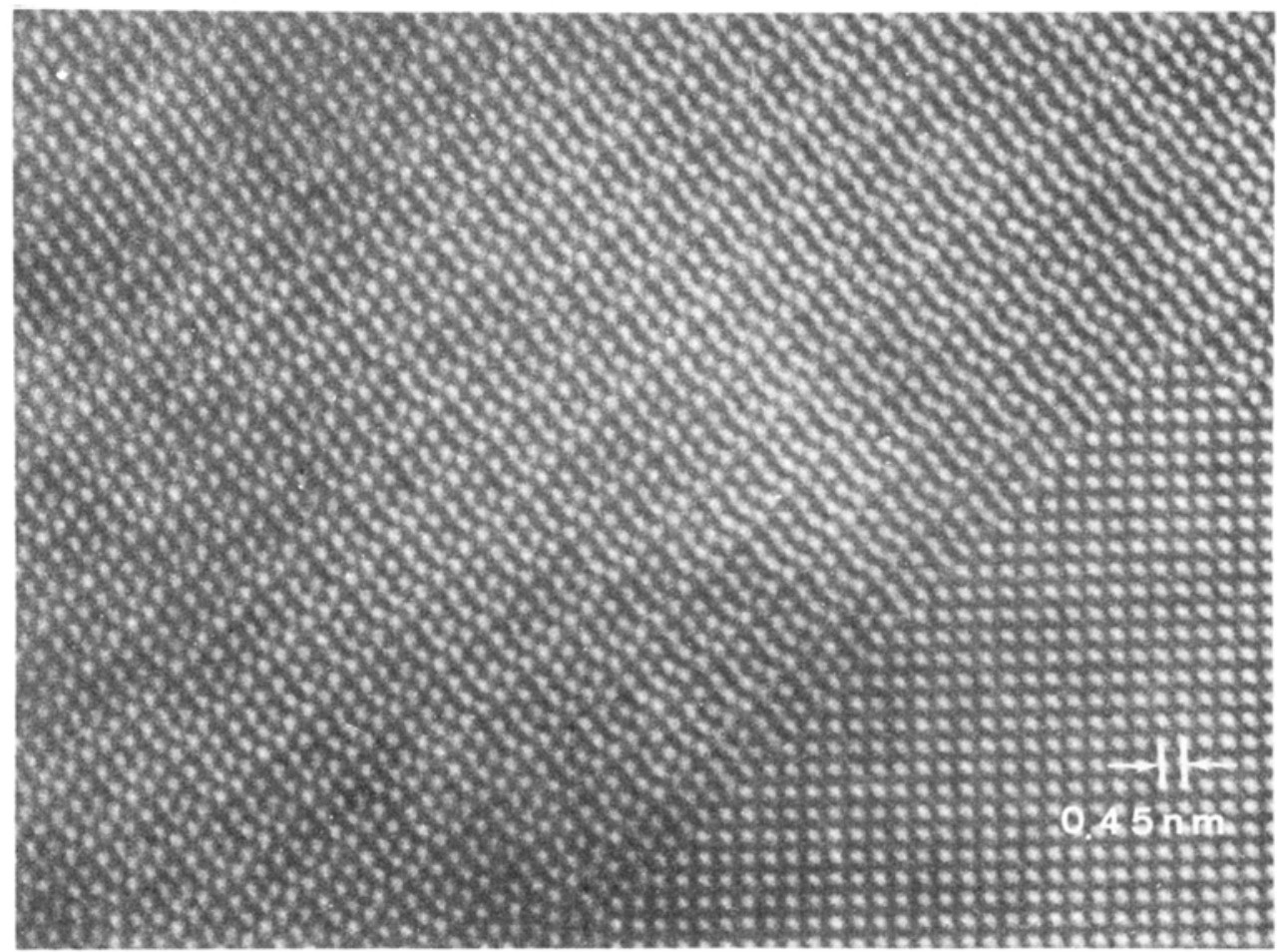

Figure 25. Tetragonal square island structure with composition $\mathrm{Au}_{3} \mathbf{M n}_{9}$ derived from the $\mathrm{Au}_{4} \mathrm{Mn}$ structure. In the right bottom corner an area of $\mathrm{Au}_{4} \mathrm{Mn}$ structure is visible. In the right top corner a small strip of one-dimensional structure is visible as well.

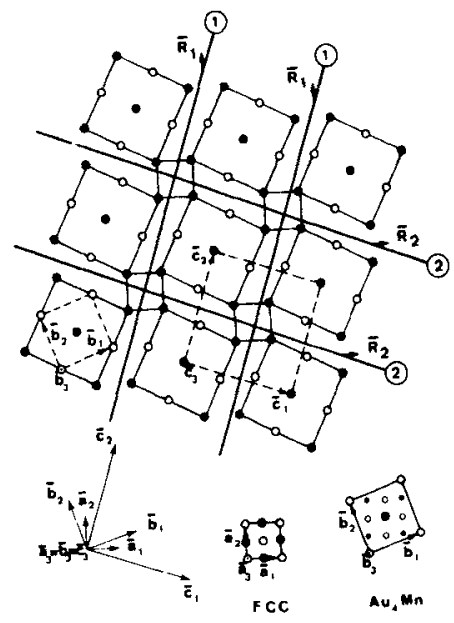

Figure 26. Model for the square island superstructure derived from the $\mathrm{Au}_{4} \mathrm{Mn}$ structure (cf. figure 25). 


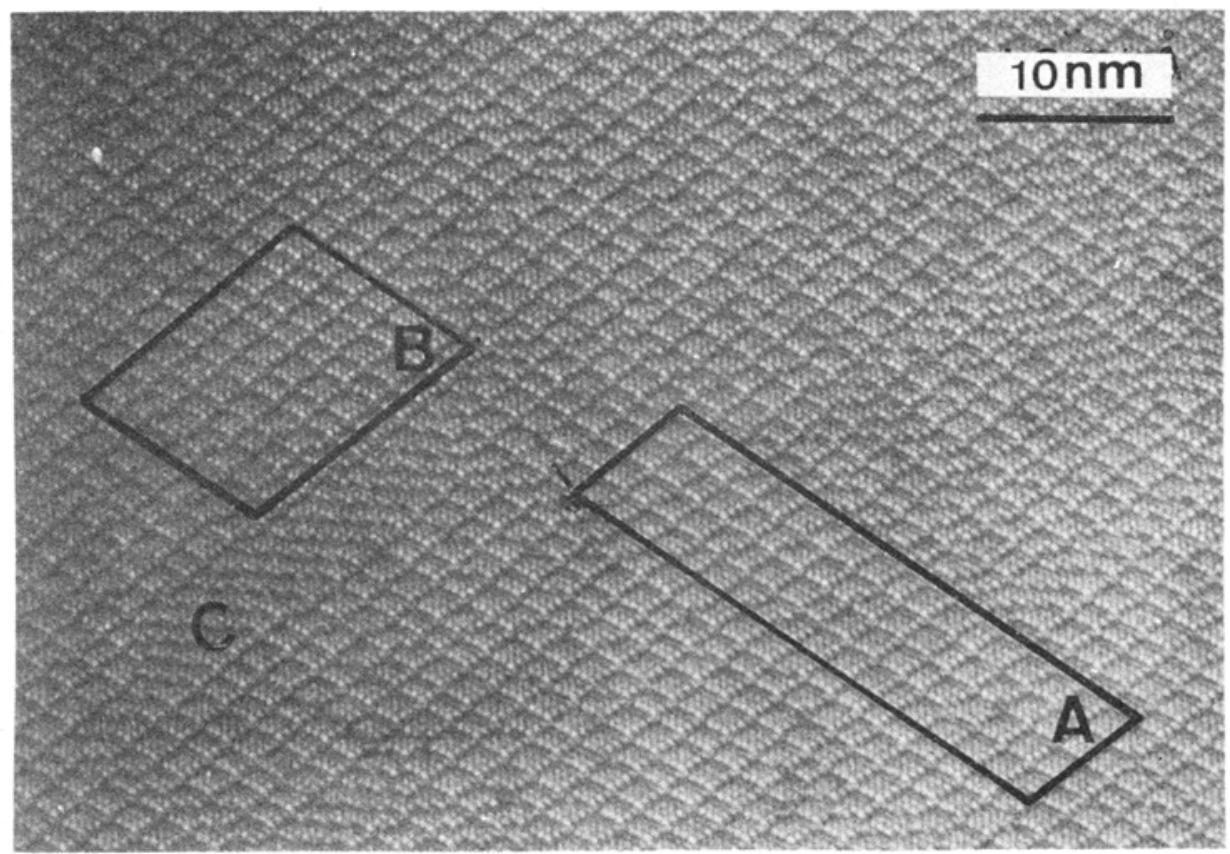

(a)

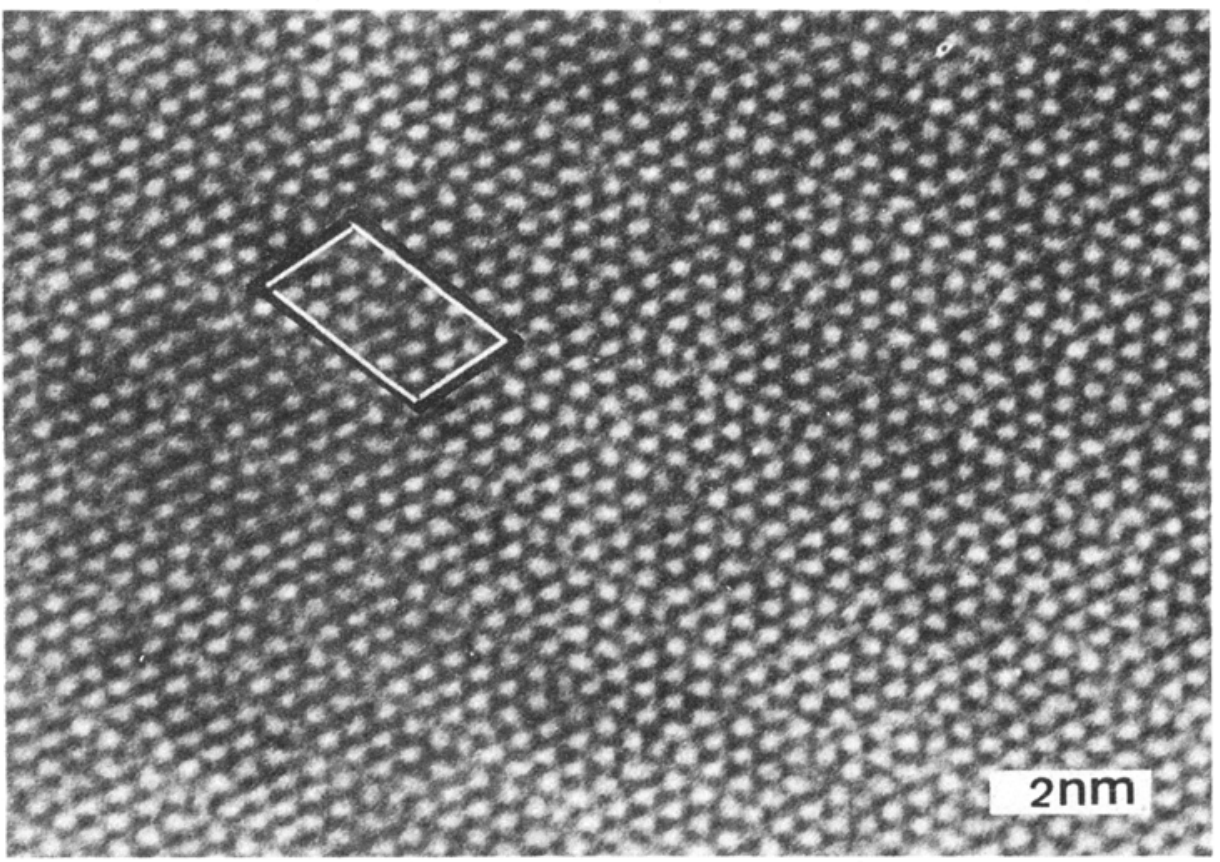

(b)

Figure 27. Parallelogram shaped island structure based on the $\mathrm{DO}_{22}$ structure. (cf. figure 12). a. superstructure dark field mode (mode 7). b. same type of structure imaged using mode 9 . 


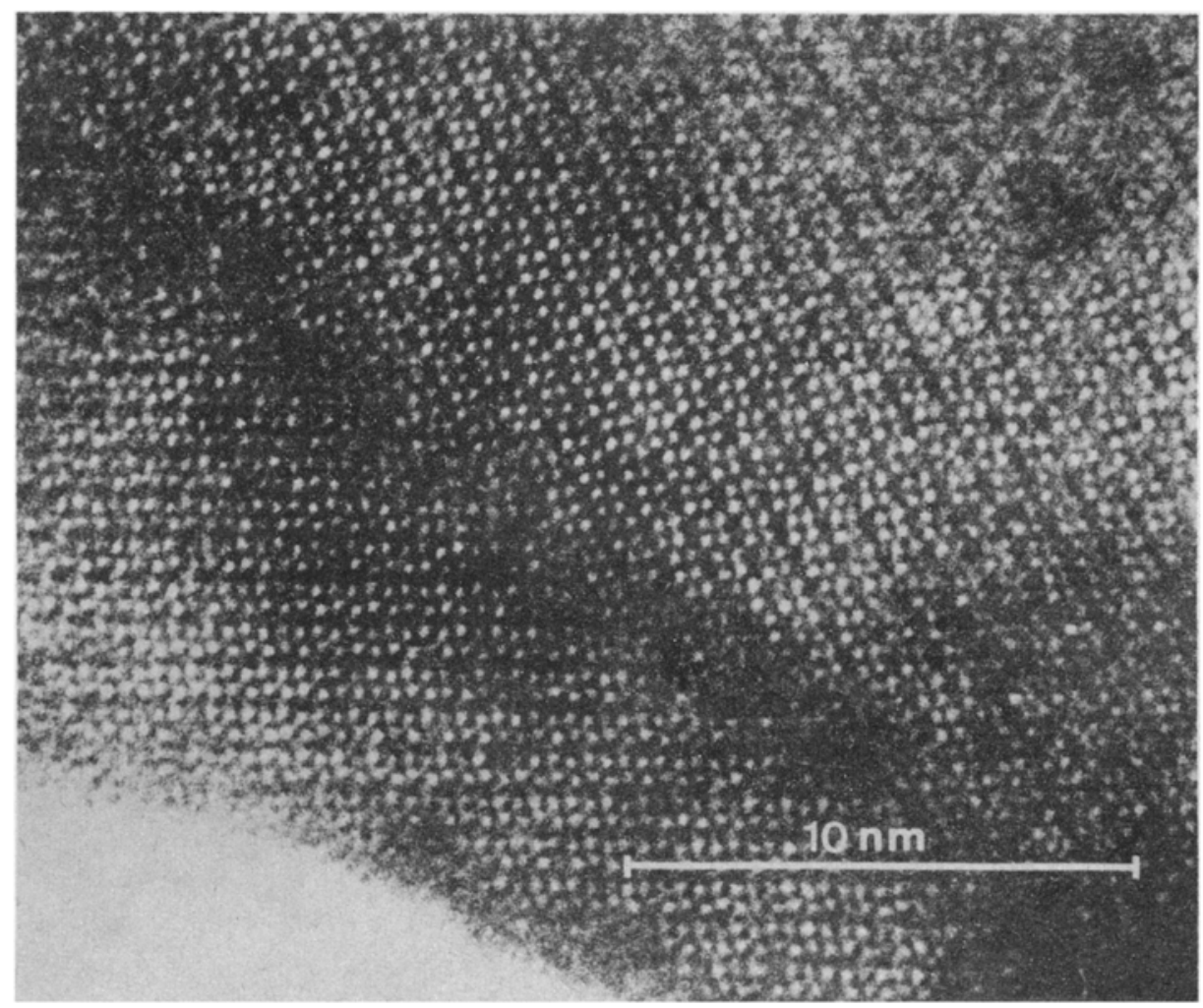

Figure 28. Monoclinic square island structure with ideal composition $\mathrm{Au}_{15} \mathrm{Mg}_{4}$. The bright dots image magnesium columns.

Figure $29 \mathrm{~b}$ is a dark field superlattice image (mode 7), where orientation as well as translation variants are quite clearly revealed. In the particular case of $\mathrm{Au}_{4} \mathrm{Mn}$ the imaging conditions are especially favourable. The eight superlattice spots due to the two variants are situated on a circle whose centre coincides with that of one square mesh of the reciprocal lattice of the basic FCC structure. It is possible to tilt the specimen in such a way that the projection of the centre of Ewald's sphere coincides with this point and moreover the objective aperture can be chosen in so as to admit only superlattice reflections. Under these conditions all image forming beams enclose the same angle with the optical axis of the microscope. As a result the angle dependent phase shifts introduced by the instrument, are same for all beams, ensuring optimum conditions for faithful image reconstruction.

The interface between coaxial variants is often inclined with respect to the common four-fold axis. Under the imaging conditions described above striking square arrays of bright dots are observed in the region of overlap (figure 30). It was demonstrated (Van Tendeloo and Amelinckx 1978) that this pattern is formed by the manganese columns which are continuous across the interface, i.e. the white dots form an image of the coincidence manganese columns. This observation supports the interpretation of the bright spots as images of manganese columns also in the other cases.

In $\mathrm{Au}_{5} \mathrm{Mn}_{2}$ the manganese columns are parallel to the two-fold axis in common to 
the four coaxial variants. The four types of domains can therefore be imaged under identical conditions. Such an image is reproduced in figure $29 \mathrm{c}$; it was made using the dark field mode collecting all superlattice spots originating from the four orientation variants situated within one mesh of the reciprocal lattice of the FCC structure (mode 7). It is clear that the majority of interfaces are coherent twins situated in cube planes. Also translation variants can be recognised. The resolution of the instrument used to make the image was not sufficient to resolve close pairs of manganese columns; these are imaged as single elongated dots. Images of this type are nevertheless sufficient to study in great detail the microtexture of this alloy, which can be formed in 84 variants within a single FCC matrix. In particular it has been possible to identify all interfaces predicted on group theoretical grounds.

In the $\mathrm{DO}_{22}$ structure of $\mathrm{Au}_{3} \mathrm{Mn}$, image in figure 29a one can distinguish two orientation variants with their c-axis in the foil plane. An antiphase boundary is present as well.

\section{Fine structure of antiphase boundaries}

As mentioned in $\$ 5.2$ all geometrically conceivable antiphase boundaries need not actually be present because the interfacial energy may be too large. In the goldmanganese system, for alloys with a composition in the vicinity of $\mathrm{Au}_{4} \mathrm{Mn}$ we noted a strong preferential orientation of the APB's in well annealed specimens; they tend to be situated in $\{110\}$ planes of the $\mathrm{Au}_{4} \mathrm{Mn}$ structure. Moreover all observed single APB's have a displacement vector which encloses with the interface normal the largest angle which is compatible with the FCC lattice i.e. they are of the type represented in the left part of figure 9.1 (a)

Such APB's are as close as possible to conservative. As represented in the same figure a change in orientation over $90^{\circ}$ causes the same APB to become strongly nonconservative. A number of such angular configurations has been observed in $\mathrm{Au}_{4} \mathrm{Mn}$ (figure 31a) in each case one leg of the angle was simple and the other one was dissociated in two components being now of the same type as the simple leg (figure $31 \mathrm{~b}$ ). This is considered to be evidence for the instability of the strongly nonconservative APB's in an alloy of this composition.

Less strongly non-conservative APB's such as the one represented in figure 9.1 (c), (left leg) and also in figure $32 a$ were found to split up in two adjacent APB's of the stable type (figure $32 \mathrm{~b}$ ) giving rise to a small strip consisting of $\mathrm{DO}_{22}$ structure.

\section{Conclusions}

The combination of high resolution electron microscopy and selected area electron diffraction has made it possible to study in great detail the micro-texture of long range ordered alloys. It is clear that this micro-texture is much more complicated than diffraction experiments alone would suggest.

High resolution electron microscopy of alloy systems present a number of characteristic features which make this method particularly suited to determine also the basic structures. By a suitable choice of the imaging conditions one can reveal preferentially the minority atoms in a binary alloy, at least along certain zones. 

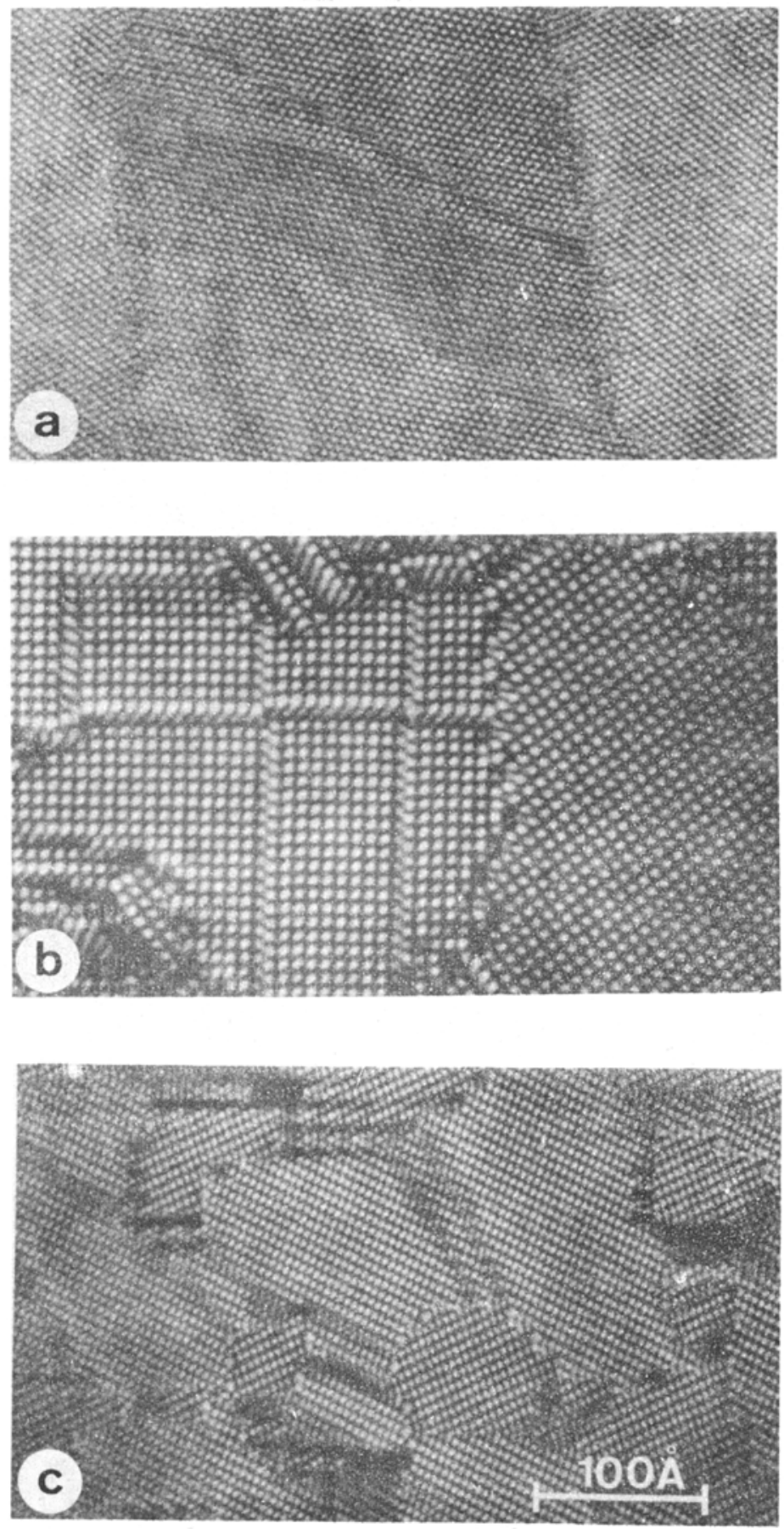

Figure 29. Domain structures in three basic structures. a. two orientation variants of the $\mathrm{DO}_{22}$ structure. The unit cells are outlined; an APB is visible as well. b. two coaxial variants of the $\mathrm{Au}_{4} \mathrm{Mn}$ structure. Also anti-phase boundaries are present. c. Several coaxial orientation variants in the $\mathrm{Au}_{5} \mathrm{Mn}_{2}$ structure; also APB's are present. 


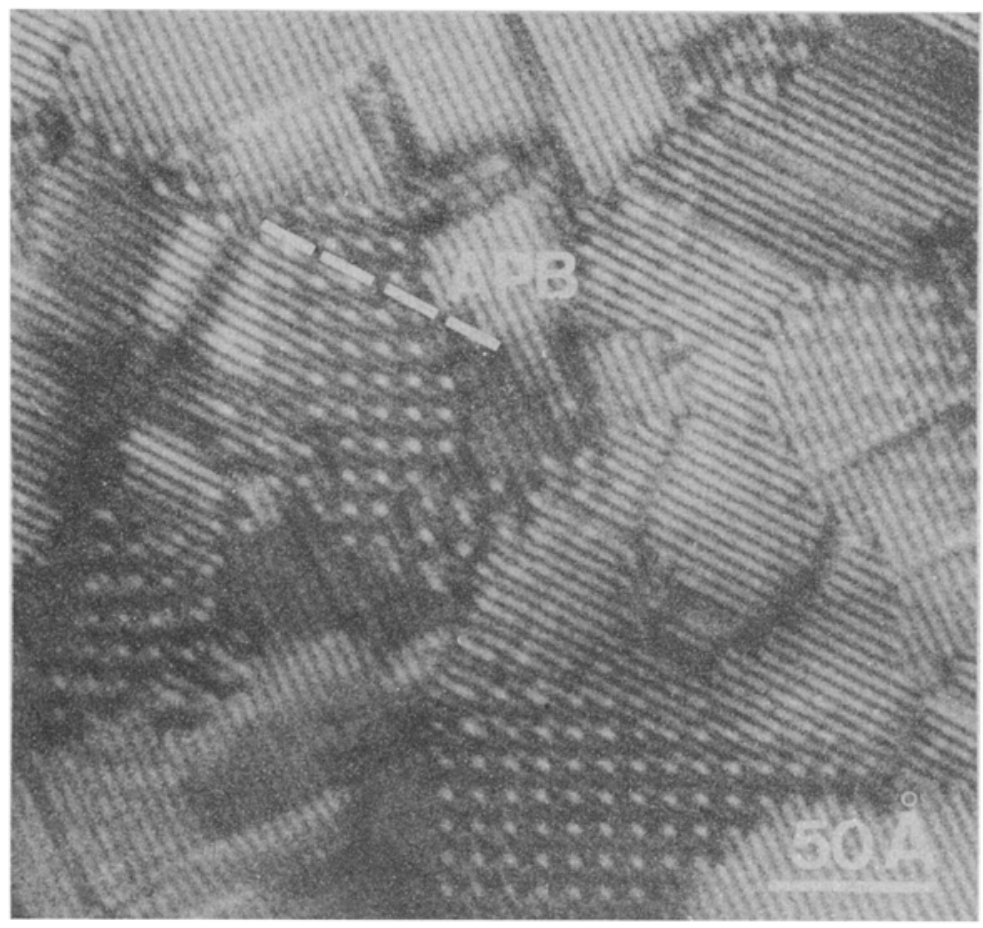

Figure 30. Pattern of coincidence columns along the inclined interface between two overlapping coaxial variants in $\mathrm{Au}_{4} \mathrm{Mn}$. The bright dots reveal the columns which are continuous across the interface.

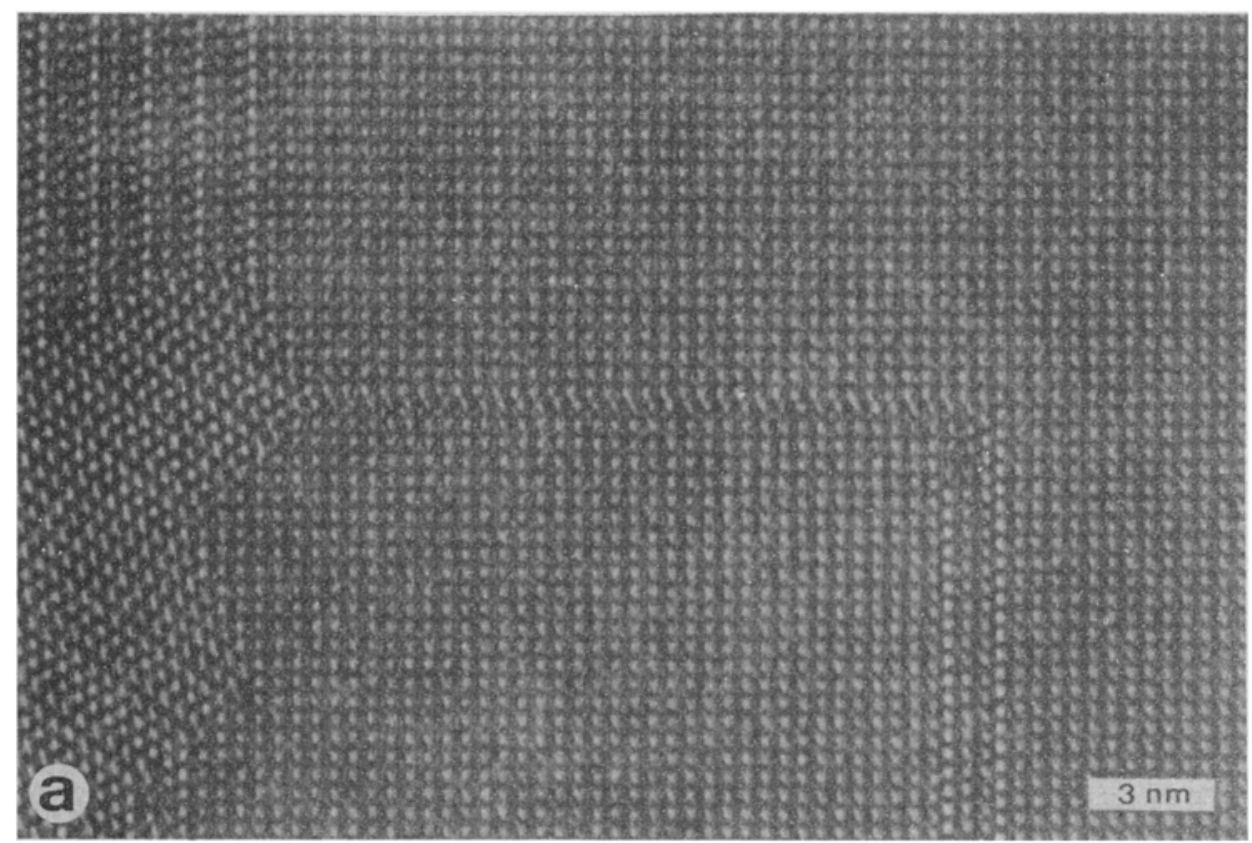

Figure 31 


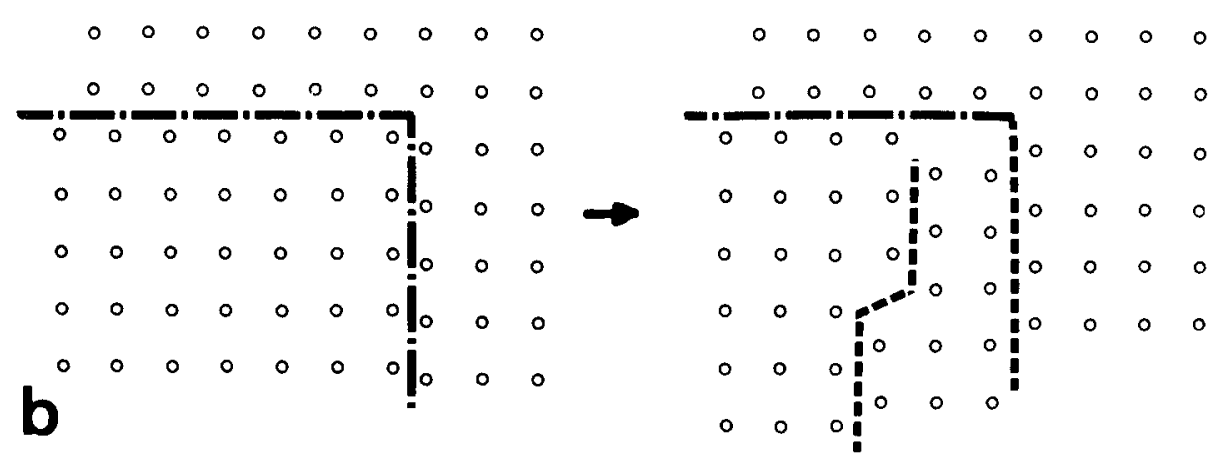

Figure 31. a. Rectangular arrangement of $A P B$ 's in $A_{4} u_{4} \mathrm{Mn}$. One "leg" is simple, the other one is dissociated in two components separated by three manganese columns. b. Analysis of the observed configuration (a) in terms of manganese columns.

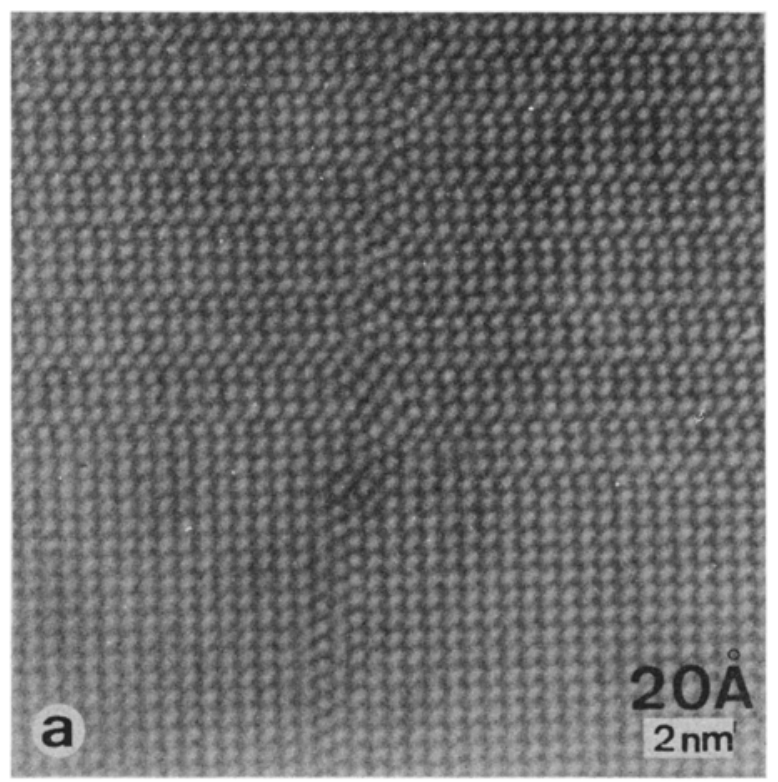

Figure 32 


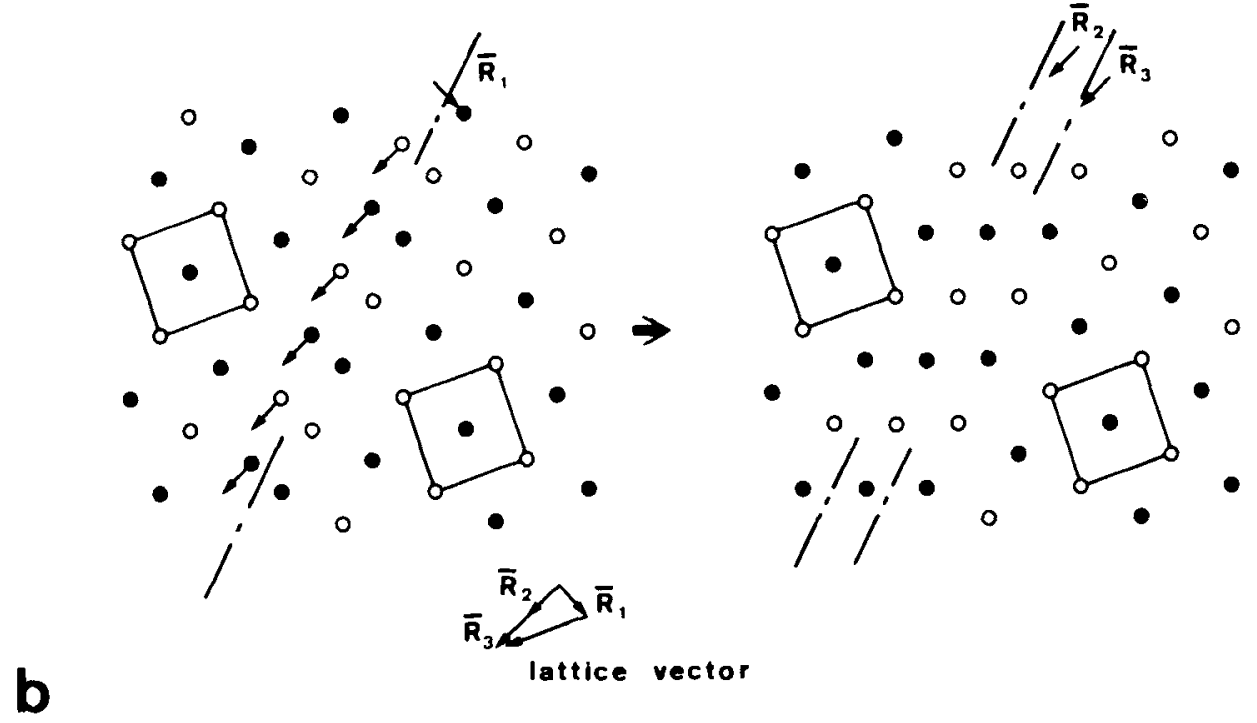

Figure 32. a. Dissociated antiphase boundary in $\mathrm{Au}_{4} \mathrm{Mn}$, intersecting a family of parallel antiphase boundaries. b. Analysis of the observed configuration a in terms of manganese columns.

A number of modulated alloy structures have been discovered first by the use of these methods.

\section{Acknowledgements}

This work was performed under the auspices of the association RUCA-SCK, with financial help from IIKW NFwo.

\section{References}

Airo P 1978 Phys. Status Solidi 47 K 107 Amelinckx S 1978-79 Chemi. Scr. 14197 Amelinckx S 1979 AIP Conf. Proc. $n=53102$

Cornellisen T, Van Tendeloo G, Van Landuyt J and Amelinckx S 1978 Phys. Status Solidi 48 K5

Cowley J M and Moodie A F 1957 Acta Crystallogr. 10609

De Ridder R, Van Landuyt J and Amelinckx S 1972 Phys. Status Solidi 9551

De Ridder R, Van Tendeloo G and Amelinckx S 1975 Phys. Stat us Solidi 30 K9

De Ridder R, Van Tendeloo G and Amelinckx S 1976 Phy's. Status Solid 33383

Den Broeder F J, Van Tendeloo G, Amelinckx S, Hornstra J, De Ridder R, Van Landuyt J and Van Daal H J 1981 Phy's. Status Solidi 67233

Fujiwara K 1957 J. Phys. Soc. Jpn 127

Fujiwara K, Hirabayashi M, Watanabe D and Ogawa S 1958 J. Phys. Soc. Jpn 13167

Gaymont M, Gratias D, Portier R and Fayard M 1977 J. Phys. C7 47

Hiraga K, Shindo D and Hirabayashi H 1980 Electron Microscopy' (eds) P Brederoo and J Van Landuyt 4170 lijima S 1975 J. Solid State Chem. 1452

Iijima S, Kimura S and Gotto M 1974 Acta Crystallogr. A30 251

Iwasaki H 1962 J. Phys. Soc. Jpn 171620 
Iwasaki H, Hirabayashi M, Fujiwara K, Watanabe D and Ogawa S 1960 J. Phys. Soc. Jpn 151771

Menter J W 1956 Proc. R. Soc. A236 119

Schubert K, Kiefer B, Wilkens M and Haufler R 1955 Z. Metallk. 46692

Scott R E 1960 J. Appl. Phys. 312112

Spence J 1981 Experimental high resolution electron microscopy (Oxford: Clarendon)

Terasaki $\mathrm{O}$ and Watanabe D 1981 Jpn J. Appl. Phys. 20381

Terasaki O and Watanabe D 1979 AJP Conf. Proc. $n^{c} 53253$

Van Dyck D 1978 Diffraction and imaging techniques in materials science (eds) S Amelinckx, R Gevers and J Van Landuyt (North Holland Publ. Co) 1355

Van Dyck D $1980 \mathrm{~J}$. Microsc. 119141

Van Landuyt J, Kohn J A, Eckart D W and Amelinckx S 1974 J. Solid State Chem. 9104

Van Landuyt J, Van Tendeloo G and Amelinckx S 1974a Phys. Status Solidi 26259

Van Landuyt J, Van Tendeloo G and Amelinckx S 1974b Phys. Status Solidi 26585

Van Landuyt J, Van Tendeloo G and Amelinckx S 1984 Phys. Status Solidi (to be published)

Van Landuyt J, Wiegers G A and Amelinckx S 1978 Phys. Status Solidi A46 479

Vanderschaeve 1980 Rev. Phys. Appl. 15711

Van Sande M, De Ridder R, Van Tendeloo G, Van Landuyt J and Amelinckx S 1978 Phys. Status Solidi A48 383

Van Tendeloo G and Amelinckx S 1974 Acta Crystallogr. A30 431

Van Tendeloo G and Amelinckx S 1977 Phys. Status Solidi A43 553, K137

Van Tendeloo G and Amelinckx S 1978a Phys. Status Solidi A47 555

Van Tendeloo G and Amelinckx S 1978b Phys. Status Solidi A49 337

Van Tendeloo G and Amelinckx S 1978c Phys. Status Solidi A50 53

Van Tendeloo G and Amelinckx S 1979 Phys. Status Solidi A51 141

Van Tendeloo G and Amelinckx S 1981 Phys. Status Solidi A65, 73, 431

Van Tendeloo G and Amelinckx S 1983 Phys. Status Solidi A (to be published)

Van Tendeloo G, De Ridder R and Amelinckx S 1978a Phys. Status Solidi A49 55

Van Tendeloo G, Den Broeder F J, Amelinckx S, De Ridder R. Van Landuyt J and Van Daal H J 1981 Phys. Status Solidi A67 217

Van Tendeloo G, Van Landuyt J and Amelinckx S 1977 Phys. Status Solidi A K137

Van Tendeloo G, Van Landuyt J and Amelinckx S 1980 Electron Microscopy (eds) P Brederoo and G Boom 1 226

Van Tendeloo G, Van Landuyt J and Amelinckx S 1983 Phys. Status Solidi A (in press)

Van Tendeloo G, Van Sande M and Amelinckx S 1979 Phys. Status Solidi A55 681

Van Tendeloo G, Wolf R, Van Dyck D and Amelinckx S 1978b Phys. Status Solidi A 105

Van Tendeloo G, Wolf R, Van Landuyt J and Amelinckx S 1978c Phys. Status Solidi A47 539

Watanabe D, Hirabayashi M and Ogawa S 1955 Acta Crystallogr. 8510

Wilkens M and Schubert K 1957 Z. Metalk. 48550

Williams P M 1976 Crystallography and Crystal Chemistry of Materials with Layered Structures (ed) F Levy (Dordrecht: Reidel Publ.) 51

Williams P M, Parry G S and Scruby C B 1974 Philos. Mag. 29695

Wilson J A, Disalvo J and Mahayan S 1974 Phys. Rev. Lett. 32882

Wilson J A, Disalvo J and Mahayan S 1975 Adv. Phys. 24117 